\title{
Determining the Regional Geochemical Background for Dissolved Trace Metals and Metalloids in Stream Waters: Protocol, Results and Limitations-The Upper Loire River Basin (France)
}

\author{
Nathalie Gassama ${ }^{1, *(D)}$, Florence Curie ${ }^{1}$, Pierre Vanhooydonck ${ }^{1}$, Xavier Bourrain ${ }^{2}$ and David Widory ${ }^{3}$ (D) \\ 1 Faculty of Sciences, University of Tours, E.A. 6392 GéoHydorsystèmes Continentaux, Parc de Grandmont, \\ 37200 Tours, France; florence.curie@univ-tours.fr (F.C.); pierre.vanhooydonck@univ-tours.fr (P.V.) \\ 2 Agence de l'Eau Loire-Bretagne, CS 36339, CEDEX 2, 45063 Orléans, France; \\ Xavier.BOURRAIN@eau-loire-bretagne.fr \\ 3 GEOTOP, Earth and Atmosphere Sciences Department, UQAM, Montréal, QC H3C 3P8, Canada; \\ widory.david@uqam.ca \\ * Correspondence: nathalie.gassama@univ-tours.fr
}

\section{check for} updates

Citation: Gassama, N.; Curie, F.; Vanhooydonck, P.; Bourrain, X.; Widory, D. Determining the Regional Geochemical Background for Dissolved Trace Metals and Metalloids in Stream Waters: Protocol, Results and Limitations-The Upper Loire River Basin (France). Water 2021, 13, 1845. https://doi.org/ 10.3390/w13131845

Academic Editor: Domenico Cicchella

Received: 22 April 2021

Accepted: 28 June 2021

Published: 1 July 2021

Publisher's Note: MDPI stays neutral with regard to jurisdictional claims in published maps and institutional affiliations.

Copyright: (c) 2021 by the authors. Licensee MDPI, Basel, Switzerland. This article is an open access article distributed under the terms and conditions of the Creative Commons Attribution (CC BY) license (https:/ / creativecommons.org/licenses/by/ $4.0 /)$.

\begin{abstract}
To avoid the improper disqualification of a watershed for which the water-rock interaction (WRI) may produce trace element concentrations exceeding established guidelines, it is of the utmost importance to properly establish natural geochemical backgrounds. Using the example of the crystalline Upper Loire River Basin, we are proposing a methodology based on the selection and chemical characterization of water and sediment samples from 10 monolithologic watersheds supposedly lowly impacted by anthropogenic inputs. We collected water samples from each watershed's spring down to its outlet and measured dissolved major, minor and selected trace elements ( $\mathrm{Al}, \mathrm{As}, \mathrm{Ba}, \mathrm{Cd}$, $\mathrm{Co}, \mathrm{Cr}, \mathrm{Cs}, \mathrm{Cu}, \mathrm{La}, \mathrm{Ni}, \mathrm{Pb}, \mathrm{U}, \mathrm{V}$ and $\mathrm{Zn}$ ) at low- and high-water stages. Results show that the chemical signature of the stream waters is controlled by mineral weatherability rather than by the available rock stock. As a result, the variability in dissolved metal concentrations between the principal lithologies is similar to that observed within each of them. While some elements mostly result from WRI, others clearly identify high inputs from topsoil leaching. Comparison with published data evidences the need to subdivide studied watersheds into distinct sectors, according to the distance from the spring, in order to define reliable natural backgrounds.
\end{abstract}

Keywords: trace metals and metalloids; stream waters; dissolved concentration; geochemical background; monolithologic watersheds

\section{Introduction}

Environmental quality standards (EQS) are generally used by regulatory institutions to evaluate the quality of stream waters at a national scale. In order to prevent the eventual disqualification of waterbodies subject to high concentrations solely resulting from natural water-rock interaction, the European Water Framework Directive (2000/60/EC [1] and 2013/39/EC [2]) proposes an approach coupling geochemical background concentrations and EQS for dissolved trace metals and metalloids (TMM). As the geogenic distribution of TMM is controlled by the occurrence of a particular lithology or of a mineral deposit, a geochemical background that has been established at a large scale (e.g., region) may not be representative of any given area independently of its geological history. Additionally, the definition of the geochemical background needs to be established before any major human activity interferes with the water chemistry of the area of interest and its vicinity. In stream waters, TMM concentrations originate from water-rock, topsoil leaching and erosion [3] and from riparian and floodplain interactions [4,5]. While some areas may not currently be impacted by anthropogenic inputs, historical human activities still have to be considered 
because of the retention of trace elements in soils and because of past and/or present atmospheric depositions [6-9]. It follows that the definition of a regional geochemical background cannot solely be based on the water-rock interaction (WRI) process but needs to integrate both the geology and historical human activities [10,11].

Previous studies have focused on establishing the geochemical background of topsoils and stream sediments at variable scales. Some have aimed at mapping trace element concentrations at national (G-Base [12]; FOREGS [13]) and regional scales [14] or for an entire ecosystem [15]. Instead of defining a geochemical background, others used geochemical thresholds to identify sites at environmental risk, using statistical analysis on large databases $[16,17]$. Finally, some choose to define the natural background at a local scale in "pristine" areas $[18,19]$. For stream waters, to our knowledge, studies intending to define their natural backgrounds are still scarce and usually correspond to premining surveys [20-22]. Studies at large or more local scales [13,23,24] highlighted the important variability of trace element concentrations in stream waters. Trace element concentrations in pristine stream waters depend on (i) the nature of the rocks and soils they are draining; (ii) the surface exchange and the exchange duration time between water and rocks, and between water and soils; (iii) the nature and quantity of atmospheric deposition stored in topsoils and (iv) the physical and biogeochemical conditions prevailing in the waterbody. For monolithologic watersheds, the geochemical signature originates from the same material all along the stream. From upstream to downstream, the concentration distribution is linked to the connections with the underlying aquifers, to the physicochemical reactions (and biological activity) occurring within the waterbody (both in the water and at the sediment/water interface) and to the surface runoff.

The Upper Loire River Basin, France (Figure 1), is characterized by crystalline rocks rich in polymetallic ores and coal deposits [25]. Its geochemical background should thus reflect trace metal anomalies associated with these mineralizations that may be considered as natural. Geological history (i.e., rock formation and hydrothermal activities associated with the ore formation) and weathering processes (rock weathering and soil formation) also have to be taken into consideration. Since the Neolithic period, the presence of these deposits favored the development of mining activities, historically followed by the implementation of metal industries and the subsequent development of large cities. It thus appears crucial to develop reliable tools to characterize the geochemical background of the Upper Loire Basin to ultimately be able to detect any eventual contamination of its stream waters.

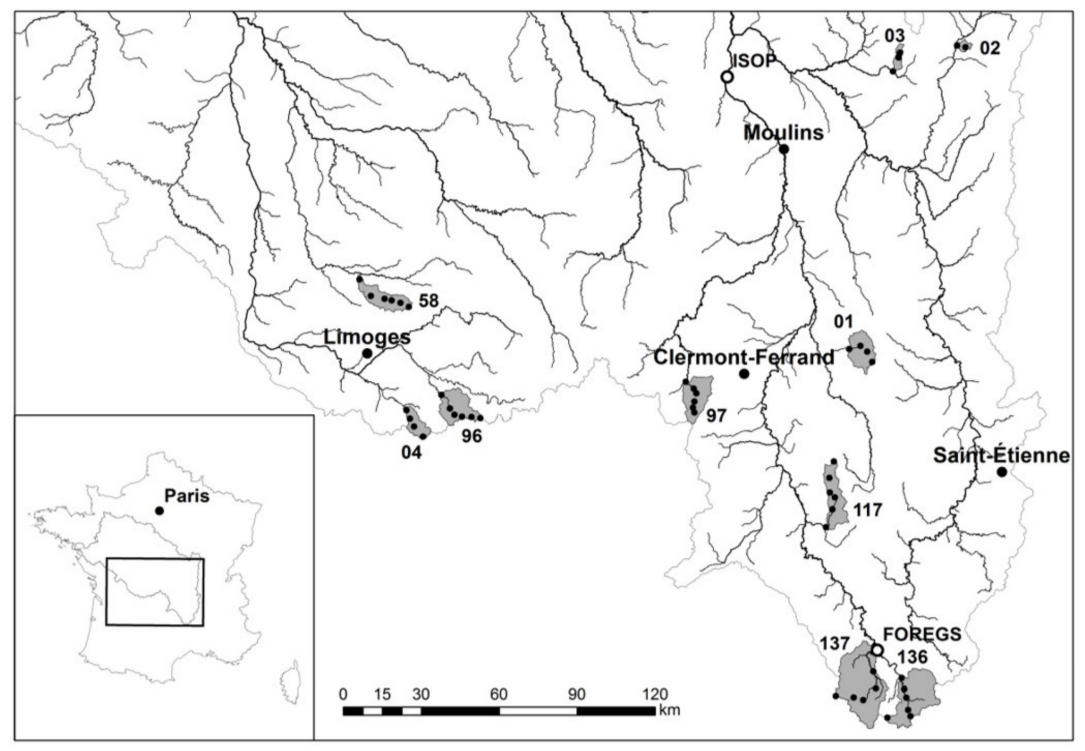

Figure 1. Locations of the selected watersheds and of the sampling sites (full circles). Open circles represent the FOREGS and ISOP Allier monitoring stations. 
Lead $(\mathrm{Pb})$ isotopes have been widely used to study drinking water supply systems [26] and groundwater circulation patterns [27] and to investigate the impact of pollution on water resources [28]. Moreover, the study of isotope systematics, such as strontium, radium or lead isotopes, demonstrated their added value for characterizing water-rock interaction (WRI) processes and for providing valuable information on the water source(s), the types of minerals water interacted with and the duration of this interaction [29]. Pb isotope ratios in water mimic those of their parent mineral hosts since dissolution and other natural processes do not fractionate them [30]. Among all of the naturally occurring $\mathrm{Pb}$ isotopes, while only ${ }^{204} \mathrm{~Pb}$ is nonradiogenic, ${ }^{206} \mathrm{~Pb},{ }^{207} \mathrm{~Pb}$ and ${ }^{208} \mathrm{~Pb}$ isotopes are daughter products from the radioactive decay of ${ }^{238} \mathrm{U},{ }^{235} \mathrm{U}$ and ${ }^{232} \mathrm{Th}$, respectively. This produces small variations in the distribution of $\mathrm{Pb}$ isotopes in nature, and these are dependent on their parent ore bodies. Thus, an approach coupling dissolved $\mathrm{Pb}$ isotope ratios and concentrations reveals itself to be a reliable approach for characterizing geochemical interactions between water and its lithology [29].

We selected several trace elements ( $\mathrm{Al}, \mathrm{As}, \mathrm{Ba}, \mathrm{Cd}, \mathrm{Co}, \mathrm{Cr}, \mathrm{Cs}, \mathrm{Cu}, \mathrm{La}, \mathrm{Ni}, \mathrm{Pb}, \mathrm{U}$, $\mathrm{V}$ and $\mathrm{Zn}$ ) representative of the diversity of TMM behaviors in aquatic systems [31]. Naturally present in granitic and gneissic rocks, As and $U$ can also be accumulated in soils on crystalline rocks [32] and aquifers [33] and, accordingly, could act as a lithology signature. Some geological formations of the studied area exhibit mineral deposits rich in $\mathrm{Ba}, \mathrm{Cu}, \mathrm{Pb}$ and $\mathrm{Zn}$. The selected elements have strong affinities for iron and manganese oxyhydroxides, but $\mathrm{Cd}$ is rather controlled by complexation with organic molecules [34] or adsorption on particulate organic matter [35]. These TMM form dissolved cations, except As, $\mathrm{Cr}$ and V, which form oxyanions. Barium is the most soluble (alkaline earth element) when Cs is weakly in the dissolved form because of high affinities for clay particles [36,37]. These TMM are directly (existing under different oxidation numbers) or/and indirectly (by sorption on Fe or Mn oxyhydroxides) controlled by redox conditions. The rare earth elements form strong complexes with phosphates [38,39] and can be tracers of phosphate fertilizer contamination [40]. We selected La, the lighter REE. Six elements (As, Cd, Cu, Cr, $\mathrm{Pb}, \mathrm{Zn}$ ) are among the UE's (European Union) priority substances [1,2].

With this in mind, the main goal of this study is to provide an operational protocol to define trace metal backgrounds in headwater streams. To fulfill this objective, we use the example of the Loire Basin, evaluating and discussing the eventual limitations of a geochemical approach based on: (i) the selection of monolithologic watersheds representative of the upper basin facies, (ii) the analysis of sediments at the springs (to determine the average upstream rock composition) and of several stream water samples to characterize the TMM concentrations as well as their distribution along the river flow and (iii) the study of lead $(\mathrm{Pb})$ isotope ratios $\left({ }^{204} \mathrm{~Pb},{ }^{206} \mathrm{~Pb},{ }^{207} \mathrm{~Pb},{ }^{208} \mathrm{~Pb}\right)$ measured in the dissolved phase of the stream waters, in sediments (average upstream geogenic signature) and in representative rocks (geogenic signature) of each watershed, as these isotope systematics have demonstrated their added value for distinguishing between the different sources of $\mathrm{Pb}$ [41-43]. Finally, we compare our TMM concentration data with those reported by previous studies in the Loire Basin [44,45], FOREGS program [13] and ISOP program [46].

\section{Regional Context}

The Loire River Basin (110,000 $\left.\mathrm{km}^{2}\right)$ covers 20\% of the French territory (Figure 1) and can be divided into three distinct parts, according to their hydrologic and geologic characteristics: the upper crystalline, the mid-sedimentary and the down crystalline basins. The upper crystalline basin (about 45,000 $\mathrm{km}^{2}$ ) is located in the Massif Central (Hercynian massif) and is characterized by various plutonic and metamorphic rocks (mainly granitic and gneissic rocks) and volcanic formations (mainly basaltic and trachytic lavas). In this part, different types of metal mineral deposits (Ag-Cu-Pb-Sn-Zn, Ag-Pb-Sb, Sn-W, $\mathrm{Au}-\mathrm{As}-\mathrm{Sb}, \mathrm{U}, \mathrm{Ba}, . .$.$) ) and coal beds are present and have been exploited. Still, most$ of the mining activities have ceased for numerous years. The upper basin hydrologic regime is pluvial with some snowmelt influences because of its continental location and 
high headwater elevation (mountainous area). The average population density is low $\left(<100\right.$ inhabitants $\left.\mathrm{km}^{-2}\right)$. The three main cities are Saint-Etienne (170,000 inhabitants), Clermont-Ferrand (142,000 inhabitants) and Limoges (134,000 inhabitants) (2015 census by the French National Institute of Statistics and Economic Studies, INSEE [47]). The headwater areas are mostly forested (forestry activity) or pastured (cattle farming) (Corine Land Cover database [48]).

\section{Methods}

\subsection{Watershed Selection}

To characterize the average rock signature of the catchment area, sediments were collected at the spring of each basin, i.e., resurgence of the underground stream, for major and trace element compositions. Stream waters were sampled from the spring down to an outlet defined according to the stream channel length and drainage area, and at two contrasting hydrologic periods (high- and low-water stages). During the high-water stage, waters originate from the stream channel but derive mainly from surface runoff. Water circulation time in the channel is short. Trace element concentrations are thus expected to be linked to the average rock signature of the drainage basin and to soil leaching: concentrations should increase with distance from the spring as the surface exchange increases. During the low-water stage, waters come from upstream via the main channel. The rock signature is thus expected to be recorded mainly at the spring but to be overprinted further downstream, as connections with different aquifers can occur as well as mixing with tributaries exhibiting distinct chemical signatures. Physicochemical reactions occurring in the stream channel and in riparian soils (such as redox reactions, complexation with organic matter, sorption on particles) control the distribution of the trace element concentrations.

The selection of 6 small monolithologic headwater streams, (i) representing the main lithologies of the Upper Loire Basin (granite, gneiss and volcanic formations) and (ii) undergoing low anthropogenic pressure, was made using the ArcGIS ${ }^{\mathrm{TM}}$ software version 9.3 (ESRI). In order to test our approach, we also included 4 small headwater streams suspected to suffer from local agriculture (mainly cattle farming) or road traffic contamination. All headwater streams were first identified using a digital terrain model with a $25 \mathrm{~m}$ resolution (BD ALTI $\left.{ }^{\circledR} 25 \mathrm{~m}, \mathrm{IGN}\right)$. The final selection was made using the following protocol. First, watersheds with a surface area $\geq 100 \mathrm{~km}^{2}$ for a stream length $>10 \mathrm{~km}$ were selected. From there, watersheds with a population density $>40$ inhab $/ \mathrm{km}^{2}$ were discarded using the 2008 census (INSEE [47]). This limit was seen as a reasonable compromise for the population density observed within the study area. According to two national databases listing polluting industries [49], watersheds that may be subject to industrial trace element contamination were excluded. Ninety-one basins remained in the selection pool at this stage. To limit the agriculture impact, watersheds with more than $50 \%$ of their land use dedicated to agriculture were rejected (selection based on the Corine Land cover [48]). This threshold is used by the Geographical Intercalibration Group to determine reference area for Central and Baltic Europe ecosystems [50]. In this area, agricultural land use mainly consists of pastures and meadows for extensive livestock farming. Using the open access Infoterre database (i.e., 1/50,000 geological maps [51]) provided by the French Geological Survey (BRGM), we created georeferenced simplified geologic maps for each of the preselected watersheds where each similar rock facies was clustered: granite-like rocks, gneiss-like rocks... This allowed the covering percentage for each lithology to be estimated. Ultimately, watersheds with a single lithology covering more than $75 \%$ of the surface area were considered as monolithologic, except for the basaltic facies for which the Sioule Basin that we selected presented the highest covering at 54\%, with an associated agriculture land use of $69 \%$. Finally, local contamination was evaluated for each selected watershed through an initial survey consisting of on-field sampling of filtered water for trace metal analysis at the outlet. Ultimately, two granitic, two gneissic, one mixed micaschist and granite and one basaltic watershed were selected as "pristine" watersheds, representative 
of the upper basin (Table 1). According to the Agence de l'Eau Loire-Bretagne (personal communication), 4 monolithologic watersheds (granitic, gneissic, rhyodacitic to andesitic cover) suspected to be impacted by agriculture practices ( 3 watersheds; cattle farming) or road traffic (1 watershed) were added at this stage.

Table 1. Characteristics of the selected watersheds: surface of the drained area, length between the spring and the outlet, main covering lithology, population and population density (between brackets, expressed in inhabitant $\mathrm{km}^{-2}$ ) and land-use.

\begin{tabular}{|c|c|c|c|c|c|c|c|c|c|}
\hline Watershed & $\begin{array}{c}\text { Surface } \\
\left(\mathrm{km}^{2}\right)\end{array}$ & $\begin{array}{l}\mathrm{L}_{\max } \\
(\mathrm{km})\end{array}$ & $\begin{array}{c}\text { Main } \\
\text { Lithology }\end{array}$ & Proportion & $\begin{array}{l}\text { Population } \\
\text { (Density) }\end{array}$ & Urban. & Agricul. & Forest & Ore \\
\hline La Durolle ${ }^{\mathrm{MW}}$ & 116 & 14 & granite & $89 \%$ & $4991(43)$ & $2 \%$ & $32 \%$ & $66 \%$ & none \\
\hline Les Echets ${ }^{\mathrm{A}}$ & 15 & 4 & granite & $81 \%$ & $984(66)$ & $2 \%$ & $68 \%$ & $30 \%$ & none \\
\hline La Couze & 116 & 30 & granite & $100 \%$ & $2338(20)$ & $1 \%$ & $37 \%$ & $58 \%$ & U-Be \\
\hline Le Chapeauroux & 396 & 54 & granite & $93 \%$ & $3544(9)$ & $0.1 \%$ & $33 \%$ & $66 \%$ & $\mathrm{U}$ \\
\hline La Petite Briance $^{\mathrm{A}}$ & 64 & 15 & gneiss & $82 \%$ & $2289(36)$ & $2 \%$ & $84 \%$ & $13 \%$ & none \\
\hline Le Doulon & 140 & 34 & gneiss & $89 \%$ & $1305(9)$ & $0.3 \%$ & $20 \%$ & $80 \%$ & none \\
\hline L'Allier & 249 & 34 & gneiss & $90 \%$ & $1775(7)$ & $0.3 \%$ & $19 \%$ & $81 \%$ & $\mathrm{Ba}-\mathrm{F}$ \\
\hline La Combade & 138 & 25 & $\begin{array}{l}\text { Micaschist } \\
+ \text { granite }\end{array}$ & $97 \%$ & $2712(20)$ & $1 \%$ & $50 \%$ & $50 \%$ & $\mathrm{Zn}-\mathrm{Cu}-\mathrm{Sn}$ \\
\hline La Sioule & 138 & 25 & Basalt & $54 \%$ & $3717(27)$ & $2 \%$ & $69 \%$ & $29 \%$ & none \\
\hline Le Richaufour ${ }^{\mathrm{A}}$ & 27 & 9 & $\begin{array}{l}\text { rhyodacite- } \\
\text { andesite }\end{array}$ & $90 \%$ & $300(11)$ & $<0.1 \%$ & $74 \%$ & $26 \%$ & none \\
\hline
\end{tabular}

Out of the 137 watersheds that corresponded to the first criterion, the final selection comprised a total of 10 watersheds complying with all criteria. For each selected river, we defined between 3 and 6 sampling sites, depending on the total river length (Table 2). Sample \#1 always corresponded to the outlet, and the sample numbers increased towards the spring, with the highest number in Table 2 corresponding to the spring itself. The geographic characteristics of the sampling sites ( $\mathrm{X}$ and $\mathrm{Y}$ coordinates, length and drained area) are given in Table S1 (Supplementary Material). Simplified geology and land cover are given in Figure 2 for two watersheds (the Allier and the Couze). 


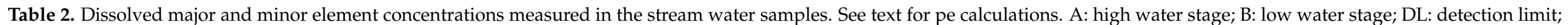
DOC: dissolved organic carbon.

\begin{tabular}{|c|c|c|c|c|c|c|c|c|c|c|c|c|c|c|c|c|c|c|c|}
\hline $\begin{array}{l}\text { Stream } \\
\text { High Water }\end{array}$ & $\begin{array}{l}\text { Label } \\
\text { urvey }\end{array}$ & $\begin{array}{l}\mathrm{T} \\
{ }^{\circ} \mathrm{C}\end{array}$ & $\mathrm{pH}$ & $\begin{array}{l}\text { TDS } \\
\mathrm{mg} / \mathrm{L}\end{array}$ & $\begin{array}{c}\mathrm{O}_{2} \\
\%\end{array}$ & $\begin{array}{c}\mathrm{HCO}_{3}^{-} \\
\mathrm{mg} / \mathrm{L}\end{array}$ & $\begin{array}{c}\mathrm{H}_{4} \mathrm{SiO}_{4} \\
\mathrm{mg} / \mathrm{L}\end{array}$ & $\begin{array}{l}\mathrm{Na}^{+} \\
\mathrm{mg} / \mathrm{L}\end{array}$ & $\begin{array}{c}\mathrm{K}^{+} \\
\mathrm{mg} / \mathrm{L}\end{array}$ & $\begin{array}{l}\mathrm{Ca}^{2+} \\
\mathrm{mg} / \mathrm{L}\end{array}$ & $\begin{array}{l}\mathrm{Mg}^{2+} \\
\mathrm{mg} / \mathrm{L}\end{array}$ & $\begin{array}{l}\mathrm{Cl}^{-} \\
\mathrm{mg} / \mathrm{L}\end{array}$ & $\begin{array}{l}\mathrm{SO}_{4}^{2-} \\
\mathrm{mg} / \mathrm{L}\end{array}$ & $\begin{array}{l}\mathrm{NO}_{3}^{-} \\
\mathrm{mg} / \mathrm{L}\end{array}$ & $\begin{array}{l}\text { Fe } \\
\text { Diss } \\
\mu \mathrm{g} / \mathrm{L}\end{array}$ & $\begin{array}{l}\text { Mn } \\
\text { Diss } \\
\mu \mathrm{g} / \mathrm{L}\end{array}$ & $\begin{array}{l}\text { DOC } \\
\mathrm{mg} / \mathrm{L}\end{array}$ & $\begin{array}{c}\text { pe } \\
\mathrm{Fe}^{2+} / \mathrm{Fe}(\mathrm{OH})_{3}\end{array}$ & $\begin{array}{c}\text { pe } \\
\mathrm{Mn}^{2+} / \text { pyrolusite }\end{array}$ \\
\hline \multirow[t]{2}{*}{ La Durolle } & $\begin{array}{l}01-1 \mathrm{~A} \\
01-2 \mathrm{~A} \\
01-3 \mathrm{~A}\end{array}$ & $\begin{array}{l}7.5 \\
7.7 \\
7.6\end{array}$ & $\begin{array}{l}7.01 \\
7.21 \\
6.90\end{array}$ & 69 & $\begin{array}{c}40 \\
100 \\
91\end{array}$ & 12.26 & 35.76 & 4.12 & 1.17 & 4.43 & 0.70 & 5.11 & 2.42 & 3.28 & 166 & 9 & 1.36 & 2.4 & 10.1 \\
\hline & $01-4 \mathrm{~A}$ & & & 68 & & 23.97 & 16.91 & 4.59 & 1.09 & 7.97 & 1.13 & 7.83 & 2.36 & 2.29 & 196 & 24 & 3.29 & & \\
\hline Les Echets & $\begin{array}{l}02-1 \mathrm{~A} \\
02-2 \mathrm{~A}\end{array}$ & 7.8 & 7.47 & 83 & 78 & 37.07 & 20.79 & 3.72 & 1.84 & 10.07 & 0.94 & 2.78 & 3.06 & 2.67 & 167 & 12 & 3.96 & 1.0 & 9.1 \\
\hline \multirow[t]{3}{*}{ La Couze } & $\begin{array}{l}02-3 \mathrm{~A} \\
58-1 \mathrm{~A} \\
58-2 \mathrm{~A} \\
58-3 \mathrm{~A}\end{array}$ & $\begin{array}{l}7.7 \\
12.3 \\
8.5\end{array}$ & $\begin{array}{l}7.18 \\
6.47 \\
7.10\end{array}$ & $\begin{array}{l}42 \\
32\end{array}$ & $\begin{array}{c}78 \\
115 \\
63\end{array}$ & $\begin{array}{l}6.60 \\
6.28\end{array}$ & $\begin{array}{l}12.56 \\
9.67\end{array}$ & $\begin{array}{l}4.67 \\
3.20\end{array}$ & $\begin{array}{l}1.49 \\
1.15\end{array}$ & $\begin{array}{l}3.07 \\
2.81\end{array}$ & $\begin{array}{l}0.71 \\
0.57\end{array}$ & $\begin{array}{l}6.54 \\
3.98\end{array}$ & $\begin{array}{l}4.32 \\
3.58\end{array}$ & $\begin{array}{l}2.41 \\
1.10\end{array}$ & $\begin{array}{l}149 \\
57\end{array}$ & $\begin{array}{c}13 \\
5\end{array}$ & $\begin{array}{l}5.35 \\
2.91\end{array}$ & $\begin{array}{l}4.1 \\
2.6\end{array}$ & $\begin{array}{l}11.1 \\
10.0\end{array}$ \\
\hline & $\begin{array}{l}58-4 \mathrm{~A} \\
58-5 \mathrm{~A}\end{array}$ & 7.5 & 6.35 & 27 & 175 & 2.81 & 14.05 & 2.21 & 0.89 & 1.32 & 0.34 & 2.63 & 1.78 & 1.38 & 114 & 13 & 3.81 & 4.6 & 11.3 \\
\hline & $58-6 \mathrm{~A}$ & 10.3 & 5.58 & 48 & 80 & 2.13 & 34.96 & 2.18 & 0.74 & 1.26 & 0.24 & 3.29 & 1.78 & 1.81 & 134 & 12 & 6.55 & 6.8 & 12.9 \\
\hline \multirow{4}{*}{$\begin{array}{c}\text { Le } \\
\text { Chapeauroux }\end{array}$} & $137-1 \mathrm{~A}$ & 6.5 & 7.75 & 52 & 39 & 15.50 & 17.80 & 3.33 & 0.97 & 4.11 & 1.04 & 3.55 & 3.16 & 2.08 & 55 & 5 & 3.14 & 0.7 & 8.7 \\
\hline & $\begin{array}{l}137-2 \mathrm{~A} \\
137-3 \mathrm{~A}\end{array}$ & $\begin{array}{l}5.8 \\
5.1\end{array}$ & $\begin{array}{l}7.42 \\
7.51\end{array}$ & 51 & $\begin{array}{l}37 \\
37\end{array}$ & 13.97 & 20.14 & 3.59 & 0.82 & 3.64 & 0.85 & 3.82 & 2.03 & 1.89 & 78 & 6 & 2.90 & 1.5 & 9.3 \\
\hline & $\begin{array}{l}137-4 \mathrm{~A} \\
137-5 \mathrm{~A}\end{array}$ & $\begin{array}{l}3.4 \\
3.2\end{array}$ & $\begin{array}{l}7.22 \\
7.02\end{array}$ & 49 & $\begin{array}{l}27 \\
27\end{array}$ & 12.36 & 21.38 & 3.26 & 0.80 & 3.33 & 0.80 & 3.67 & 1.91 & 1.81 & - & 14 & 2.73 & 1.2 & 9.5 \\
\hline & $137-6 \mathrm{~A}$ & 2.1 & 6.30 & 35 & 30 & 12.68 & 15.54 & 1.31 & 0.41 & 1.27 & 0.35 & 1.12 & 1.71 & 0.94 & 103 & 3 & 2.36 & 4.7 & 11.7 \\
\hline $\begin{array}{l}\text { La Petite } \\
\text { Briance }\end{array}$ & $\begin{array}{l}04-2 \mathrm{~A} \\
04-3 \mathrm{~A} \\
04-4 \mathrm{~A}\end{array}$ & & & & & & & & & & & & & & & & & & \\
\hline \multirow[t]{3}{*}{ Le Doulon } & $\begin{array}{l}117-1 \mathrm{~A} \\
117-2 \mathrm{~A} \\
117-3 \mathrm{~A}\end{array}$ & $\begin{array}{l}8.3 \\
7.6 \\
7.6\end{array}$ & $\begin{array}{l}7.69 \\
7.61 \\
7.30\end{array}$ & $\begin{array}{l}81 \\
64\end{array}$ & $\begin{array}{l}53 \\
48 \\
53\end{array}$ & $\begin{array}{l}28.50 \\
22.52\end{array}$ & $\begin{array}{l}32.76 \\
23.26\end{array}$ & $\begin{array}{l}3.75 \\
4.61\end{array}$ & $\begin{array}{l}1.22 \\
1.04\end{array}$ & $\begin{array}{l}5.14 \\
3.78\end{array}$ & $\begin{array}{l}1.58 \\
1.09\end{array}$ & $\begin{array}{l}3.33 \\
3.12\end{array}$ & $\begin{array}{l}3.15 \\
2.95\end{array}$ & $\begin{array}{l}1.64 \\
1.74\end{array}$ & $\begin{array}{l}187 \\
113\end{array}$ & $\begin{array}{l}2 \\
3\end{array}$ & $\begin{array}{l}2.30 \\
2.49\end{array}$ & $\begin{array}{l}0.3 \\
0.8\end{array}$ & $\begin{array}{l}9.0 \\
9.1\end{array}$ \\
\hline & $\begin{array}{l}117-4 \mathrm{~A} \\
117-5 \mathrm{~A}\end{array}$ & $\begin{array}{l}8.3 \\
7.8\end{array}$ & $\begin{array}{l}7.43 \\
7.23\end{array}$ & 68 & $\begin{array}{l}48 \\
42\end{array}$ & 17.81 & 30.87 & 3.52 & 1.08 & 4.32 & 1.00 & 4.15 & 2.72 & 2.08 & 59 & 6 & 2.54 & 1.6 & 9.3 \\
\hline & $117-6 \mathrm{~A}$ & 5.3 & 6.69 & 37 & 33 & 5.59 & 22.19 & 1.75 & 0.34 & 1.55 & 0.42 & 1.37 & 2.28 & 1.51 & 192 & 8 & 5.33 & 3.3 & 10.7 \\
\hline \multirow[t]{2}{*}{ L'Allier } & $\begin{array}{l}136-1 \mathrm{~A} \\
136-2 \mathrm{~A} \\
136-3 \mathrm{~A} \\
136-4 \mathrm{~A} \\
136-5 \mathrm{~A}\end{array}$ & $\begin{array}{l}6.9 \\
8.0 \\
7.7 \\
5.5 \\
5.7\end{array}$ & $\begin{array}{l}7.57 \\
7.26 \\
7.23 \\
6.98 \\
7.17\end{array}$ & 31 & $\begin{array}{l}37 \\
32 \\
50 \\
29 \\
38\end{array}$ & 6.95 & 12.42 & 2.03 & 0.45 & 2.22 & 0.61 & 2.91 & 2.22 & 1.57 & 74 & 4 & 0.64 & 1.1 & 9.1 \\
\hline & $136-6 \mathrm{~A}$ & 5.2 & 6.43 & 22 & 30 & 2.04 & 13.92 & 0.73 & 0.28 & 0.97 & 0.32 & 0.96 & 2.14 & 0.82 & 14 & 3 & $<\mathrm{DL}$ & 5.2 & 11.5 \\
\hline
\end{tabular}


Table 2. Cont.

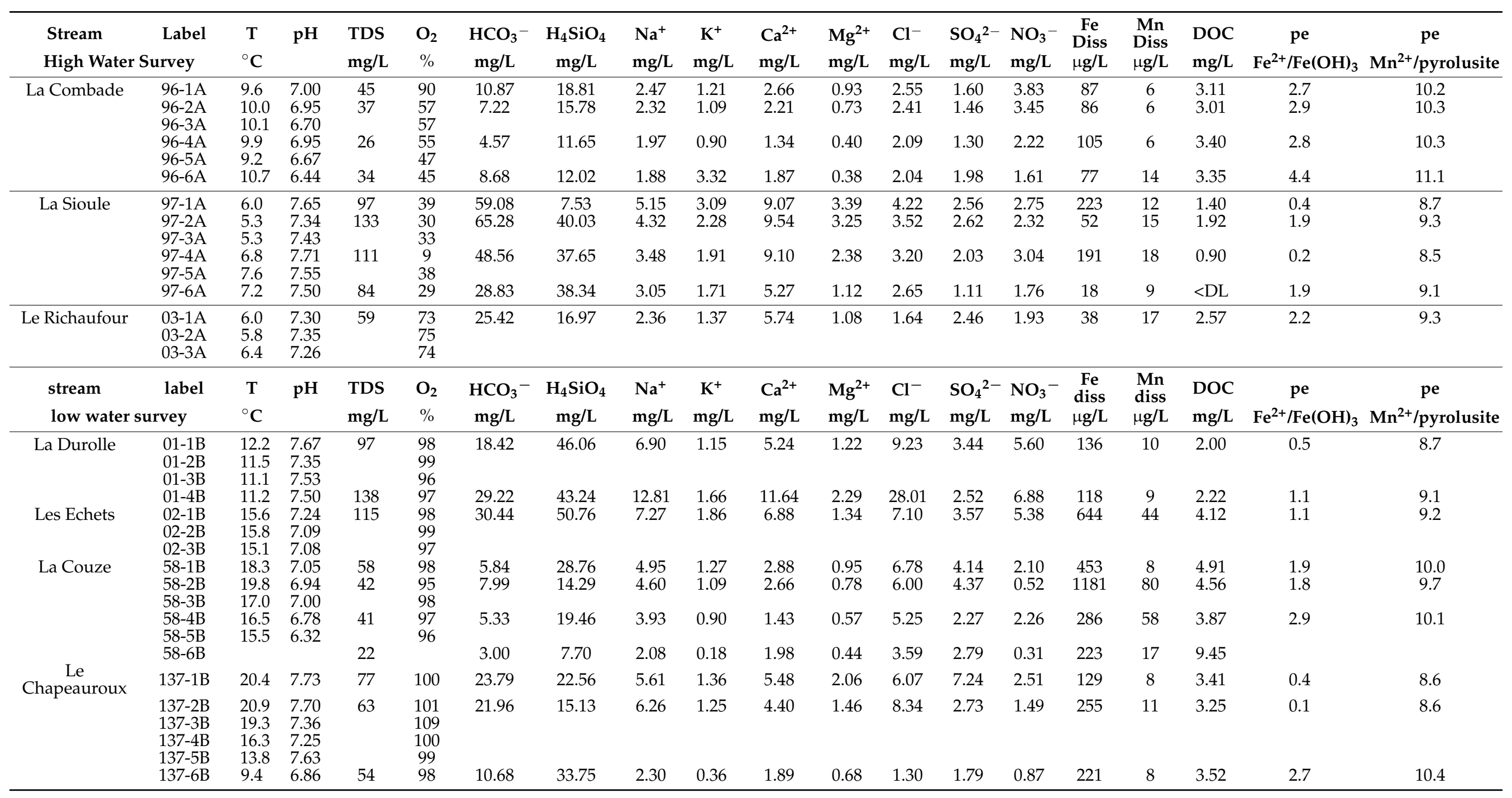


Table 2. Cont

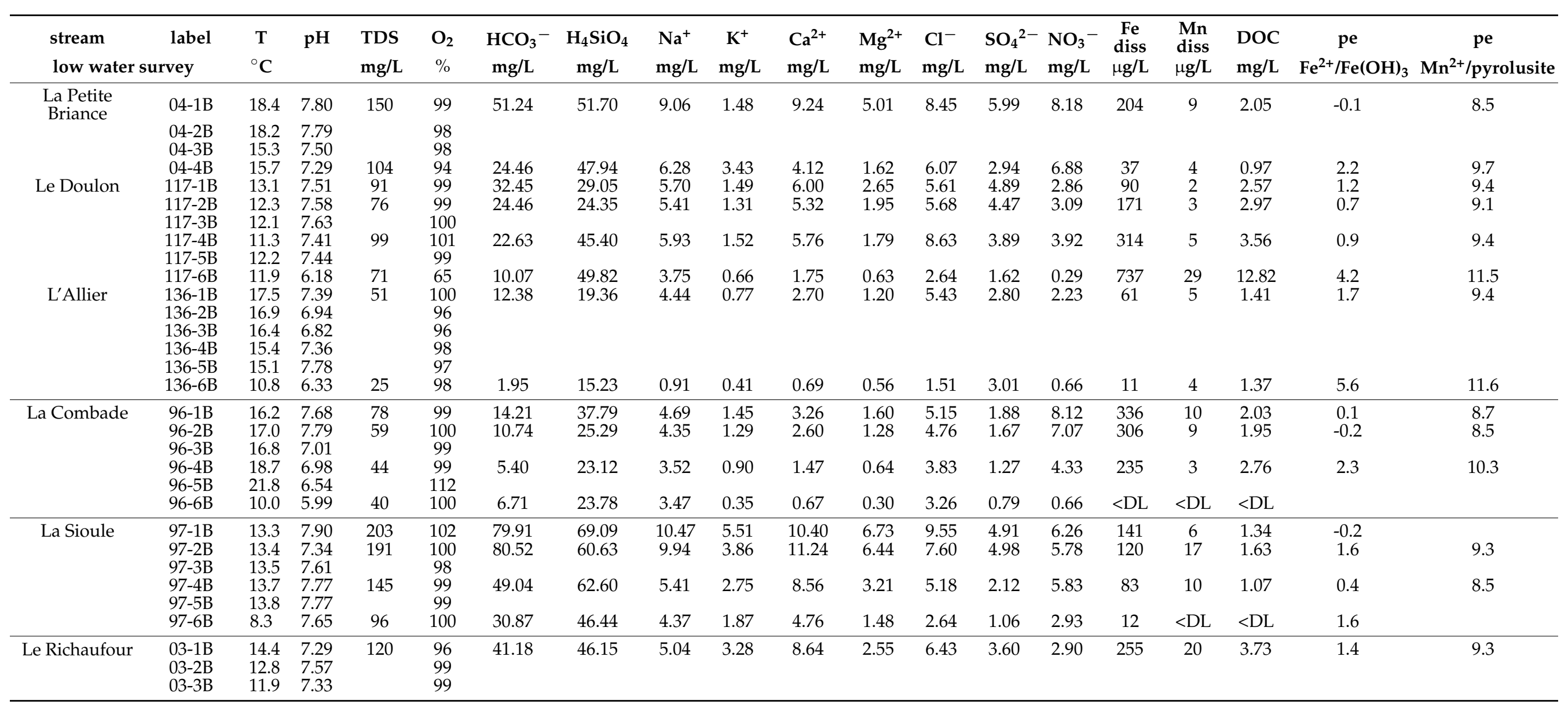



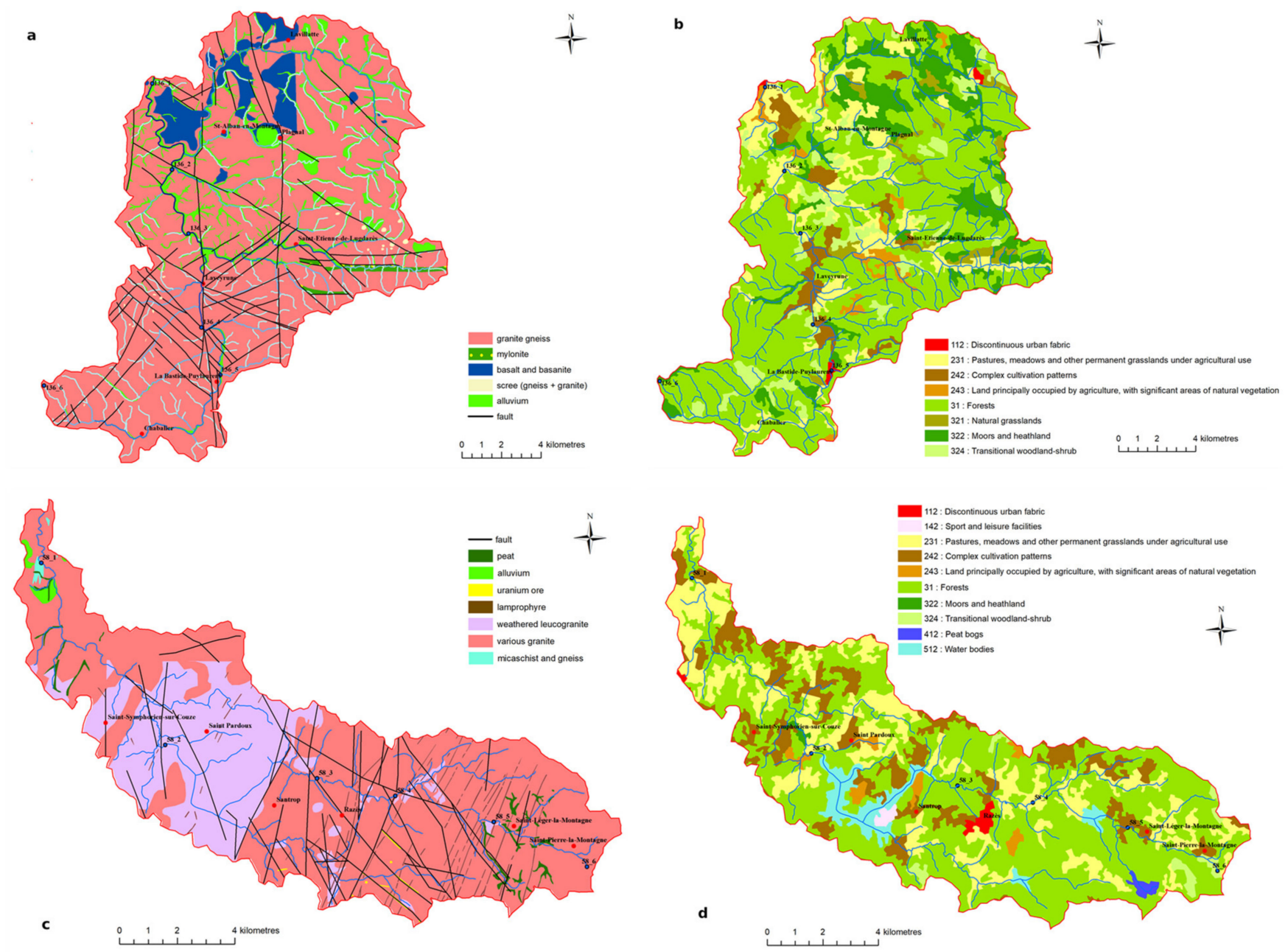

Figure 2. The Allier watershed: (a) simplified geological map; (b) land-cover. The Couze watershed: (c) simplified geological map [51]; (d) land cover [48]. The standard CLC colors were modified for clarity.

\subsection{Materials and Methods}

\subsubsection{Sampling and Analytical Chemistry}

We used the HYDRO-MEDDE/DE [52] hydrological database, available from the French regulatory institution Eaufrance, to determine the most accurate high- and lowwater stage sampling periods. Statistical calculations were made on data collected over the 396 monitoring stations of the Upper basin during the last 30 years for the high-water stage and during the last 10 years for the low-water stage. Maximum peak flows were recorded from February to April and low ones in August. Due to the geographical locations of the selected watersheds (head waters), we selected the following periods for the high and low flow surveys: March to April 2015 and mid-July 2016, respectively. For each stream water, water samples were collected from bridges in the middle of the river channel, except for spring samples. Springs were sampled after the different water outlets formed a stream channel. To determine reaction conditions controlling the trace element speciation, electrical conductivity, $\mathrm{pH}$, temperature and dissolved oxygen were measured in situ in the stream. Major cations and anions, dissolved silica, dissolved organic carbon (DOC), $\mathrm{Fe}, \mathrm{Mn}$ and selected trace elements ( $\mathrm{Al}, \mathrm{As}, \mathrm{Ba}, \mathrm{Cs}, \mathrm{Cd}, \mathrm{Co}, \mathrm{Cu}, \mathrm{Cr}, \mathrm{La}, \mathrm{Ni}, \mathrm{Pb}, \mathrm{U}, \mathrm{V}, \mathrm{Zn}$ ) were also analyzed in filtered waters. Samples were filtered on site through $0.45 \mu \mathrm{m}$ cellulose acetate membrane filters that were first rinsed with the sample using a hand pump. Aliquots 
for major cations, $\mathrm{DOC}, \mathrm{Mn}$ and $\mathrm{Fe}$, and for trace elements were acidified to $\mathrm{pH}=2$ with Suprapur ${ }^{\circledR}$ grade nitric acid (Merck). Samples were collected in polypropylene bottles previously washed with the same nitric acid and stored at about $4{ }^{\circ} \mathrm{C}$ until further analysis. Alkalinity was measured by titrimetry (Gran method calculation) on the day of sampling. All standard solutions were certified (Certipur ${ }^{\circledR}$ Merck). Each analysis of 10-sample batches was bracketed between two standard calibration runs. Ionic balances ranged from 2 to $13 \%$ for the less mineralized waters. Major cation $(\mathrm{Na}, \mathrm{K}, \mathrm{Ca}, \mathrm{Mg})$ concentrations were determined by atomic absorption or emission flame spectrometry (detection limit $=0.04 \mathrm{mg} \mathrm{L}^{-1}$ for $\mathrm{K}, 0.23 \mathrm{mg} \mathrm{L}^{-1}$ for $\mathrm{Na}, 0.40 \mathrm{mg} \mathrm{L}^{-1}$ for $\mathrm{Ca}$ and $0.24 \mathrm{mg} \mathrm{L}^{-1}$ for $\left.\mathrm{Mg}\right)$, and anion $\left(\mathrm{Cl}^{-}\right.$, $\mathrm{SO}_{4}{ }^{2-}$ and $\mathrm{NO}_{3}{ }^{-}$) concentrations by ionic chromatography using a chemical suppressor (detection limit $=0.71 \mathrm{mg} \mathrm{L}^{-1}$ for $\mathrm{Cl}^{-}, 1.92 \mathrm{mg} \mathrm{L}^{-1}$ for $\mathrm{SO}_{4}{ }^{2-}$ and $1.24 \mathrm{mg} \mathrm{L}^{-1}$ for $\mathrm{NO}_{3}{ }^{-}$). $\mathrm{H}_{4} \mathrm{SiO}_{4}$ was measured by colorimetry (detection limit $=1.88 \mathrm{mg} \mathrm{L}^{-1}$ ). Typical measurement uncertainties were $\pm 5 \%$ for major elements. DOC was determined on a Shimadzu TOC/TN analyzer via a nonpurgeable organic carbon (NPOC) measurement that involved acidification, sparging (inorganic carbon removal) and combustion of the sample at $680^{\circ} \mathrm{C}$. The accuracy of DOC concentration measurements was $\pm 0.1 \mathrm{mg} \mathrm{L}^{-1}$, estimated on blank samples and standards with a detection limit of $0.5 \mathrm{mg} \mathrm{L}^{-1}$. Due to an instrument failure during the project, Fe and Mn concentrations had to be measured with distinct techniques. Fe and $\mathrm{Mn}$ in high-water samples were determined by GFAAS, and in low-water samples by ICP-MS (Laboratoire Départemental de Touraine, Tours, France). Some of the low-water samples were also analyzed by GFAAS, allowing accuracy between the two techniques to be crosschecked: deviations were calculated $<10 \%$. For the analysis by GFAAS, the detection limits were $2.2 \mu \mathrm{g} \mathrm{L}^{-1}$ for Fe and $1.6 \mu \mathrm{g} \mathrm{L}^{-1}$ for Mn, and by ICP-MS, they were $5.0 \mu \mathrm{g} \mathrm{L}^{-1}$ and $1.1 \mu \mathrm{g} \mathrm{L}^{-1}$, respectively. Analytical results are presented in Table 2. Trace element analysis was performed by the SARM laboratory (SARM-CRPG UMR 7358, Nancy, France). Accuracy and precision of determinations were checked at regular intervals with certified reference materials (NRC-CNRC SLRS-6). Detection limits and accuracy on trace element measurements are presented in Table 3. Table 4 reports concentrations for the different trace elements as well as the corresponding EQS values $[53,54]$.

Table 3. Detection limits and accuracy on measurements of trace element concentrations.

\begin{tabular}{|c|c|c|c|c|c|c|c|c|c|c|c|c|c|c|c|}
\hline & & $\mathbf{A} 1^{*}$ & As & $\mathrm{Ba}$ & $\mathrm{Cd}$ & Co & $\mathrm{Cr}$ & Cs & $\mathrm{Cu}$ & $\mathrm{Ni}$ & $\mathrm{Pb}$ & $\mathbf{U}$ & $\mathbf{V}$ & $\mathrm{Zn}$ & La \\
\hline $\begin{array}{l}\text { Accuracy }(\%) \\
\text { according to } \\
\text { concentration }\end{array}$ & $\begin{array}{c}>100 \mu \mathrm{g} / \mathrm{L} \\
>10 \mu \mathrm{g} / \mathrm{L} \\
>1 \mu \mathrm{g} / \mathrm{L} \\
>0.1 \mu \mathrm{g} / \mathrm{L} \\
>0.01 \mu \mathrm{g} / \mathrm{L} \\
>100 \mathrm{ng} / \mathrm{L} \\
>10 \mathrm{ng} / \mathrm{L} \\
>1 \mathrm{ng} / \mathrm{L}\end{array}$ & $\begin{array}{l}<5 \% \\
<15 \% \\
* *\end{array}$ & $\begin{array}{l}<5 \% \\
<10 \% \\
<15 \% \\
* *\end{array}$ & $\begin{array}{l}<5 \% \\
<10 \% \\
<20 \% \\
* *\end{array}$ & $\begin{array}{l}<5 \% \\
<10 \% \\
<20 \% \\
* *\end{array}$ & $\begin{array}{l}<5 \% \\
<10 \% \\
<20 \% \\
* *\end{array}$ & $\begin{array}{l}<5 \% \\
<15 \% \\
<20 \% \\
* *\end{array}$ & $\begin{array}{l}<5 \% \\
<10 \% \\
* *\end{array}$ & $\begin{array}{l}<5 \% \\
<15 \% \\
<20 \% \\
* *\end{array}$ & $\begin{array}{l}<5 \% \\
<10 \% \\
<20 \% \\
* *\end{array}$ & $\begin{array}{l}<5 \% \\
<10 \% \\
* *\end{array}$ & $\begin{array}{l}<5 \% \\
<10 \% \\
<20 \% \\
* *\end{array}$ & $\begin{array}{l}<5 \% \\
<15 \% \\
* *\end{array}$ & $\begin{array}{l}<10 \% \\
<20 \% \\
* *\end{array}$ & $\begin{array}{l} \\
<10 \% \\
<15 \% \\
* *\end{array}$ \\
\hline $\begin{array}{l}\text { Detection } \\
\text { limit }\end{array}$ & $\begin{array}{l}\text { DL } \mu g / L \\
D L n g / L\end{array}$ & 0.60 & 0.025 & 0.003 & 0.005 & 0.005 & 0.04 & 0.001 & 0.01 & 0.01 & 0.002 & 0.001 & 0.015 & 0.015 & 0.3 \\
\hline
\end{tabular}


Table 4. Dissolved trace element concentrations (expressed in $\mu \mathrm{g} \mathrm{L}^{-1}$ ). A: high waters; B: low waters; DL: detection limit. For a given element, extreme values are highlighted (light grey: minimum; rectangle: maximum).

\begin{tabular}{|c|c|c|c|c|c|c|c|c|c|c|c|c|c|c|}
\hline Label & $\mathrm{Al}$ & As & Ba & $\mathrm{Cd}$ & Co & $\mathrm{Cr}$ & Cs & $\mathrm{Cu}$ & $\mathbf{N i}$ & $\mathbf{P b}$ & $\mathbf{U}$ & V & $\mathrm{Zn}$ & La \\
\hline $01-1 \mathrm{~A}$ & 30.6 & 0.651 & 10.84 & 0.032 & 0.059 & 0.45 & 0.044 & 1.00 & 0.56 & 0.237 & 0.251 & 0.190 & 2.30 & 0.220 \\
\hline $01-2 \mathrm{~A}$ & 66.5 & 0.546 & 10.06 & 0.024 & 0.047 & 0.16 & 0.033 & 0.86 & 0.14 & 0.125 & 0.224 & 0.159 & 6.13 & 0.189 \\
\hline $01-3 \mathrm{~A}$ & 46.4 & 0.382 & 12.89 & 0.022 & 0.051 & 0.13 & 0.042 & 0.60 & 0.14 & 0.131 & 0.183 & 0.160 & 1.28 & 0.184 \\
\hline $01-4 \mathrm{~A}$ & 25.9 & 0.489 & 14.83 & 0.019 & 0.131 & 0.18 & 0.035 & 0.79 & 0.25 & 0.193 & 0.071 & 0.164 & 2.15 & 0.187 \\
\hline $02-1 \mathrm{~A}$ & 297 & 1.345 & 162.7 & 0.024 & 0.273 & 0.59 & 0.255 & 1.48 & 0.90 & 0.630 & 0.171 & 0.865 & 5.43 & 0.230 \\
\hline $02-3 \mathrm{~A}$ & 174 & 0.714 & 116.2 & 0.038 & 0.129 & 0.33 & 0.439 & 1.11 & 0.37 & 0.369 & 0.175 & 0.454 & 7.13 & 0.086 \\
\hline $58-1 \mathrm{~A}$ & 72.5 & 0.572 & 31.32 & 0.025 & 0.123 & 0.21 & 0.077 & 1.23 & 0.48 & 0.362 & 0.935 & 0.225 & 3.34 & 0.114 \\
\hline $58-2 \mathrm{~A}$ & 35.2 & 0.388 & 24.63 & 0.005 & 0.033 & 0.18 & 0.076 & 0.56 & 0.20 & 0.056 & 1.22 & 0.088 & 2.58 & 0.143 \\
\hline $58-3 \mathrm{~A}$ & 178 & 0.640 & 20.66 & 0.027 & 0.166 & 0.28 & 0.133 & 1.01 & 0.31 & 0.188 & 1.34 & 0.218 & 7.25 & 0.333 \\
\hline $58-4 \mathrm{~A}$ & 101 & 0.484 & 9.70 & 0.027 & 0.097 & 0.15 & 0.151 & 0.50 & 0.32 & 0.223 & 1.27 & 0.139 & 1.55 & 0.095 \\
\hline $58-5 \mathrm{~A}$ & 200 & 0.590 & 7.36 & 0.027 & 0.130 & 0.22 & 0.161 & 0.86 & 0.23 & 0.256 & 0.334 & 0.279 & 4.55 & 0.141 \\
\hline $58-6 \mathrm{~A}$ & 245 & 0.815 & 7.66 & 0.035 & 0.140 & 0.30 & 0.179 & 1.57 & 0.24 & 0.379 & 0.416 & 0.483 & 6.54 & 0.210 \\
\hline $137-1 \mathrm{~A}$ & 110 & 1.08 & 16.88 & 0.019 & 0.094 & 0.35 & 0.073 & 1.16 & 0.80 & 0.131 & 1.14 & 0.353 & 8.10 & 0.174 \\
\hline $137-2 \mathrm{~A}$ & 56.1 & 0.949 & 24.64 & 0.016 & 0.067 & 0.27 & 0.077 & 0.59 & 0.23 & 0.102 & 0.233 & 0.301 & 0.78 & 0.146 \\
\hline $137-3 \mathrm{~A}$ & 52.8 & 0.954 & 23.16 & 0.021 & 0.079 & 0.28 & 0.071 & 0.40 & 0.22 & 0.123 & & & 1.02 & 0.143 \\
\hline $137-4 \mathrm{~A}$ & 117 & 0.893 & 27.63 & 0.028 & 0.095 & 0.30 & 0.084 & 0.91 & 0.23 & 0.135 & 0.255 & 0.298 & 9.91 & 0.138 \\
\hline $137-5 \mathrm{~A}$ & 91.5 & 0.962 & 16.26 & 0.029 & 0.048 & 0.38 & & 0.49 & 0.16 & 0.178 & & & 5.43 & 0.103 \\
\hline $137-6 \mathrm{~A}$ & 107 & 0.620 & 8.94 & 0.047 & 0.046 & 0.25 & 0.093 & 0.46 & 0.15 & 2.63 & 0.050 & 0.245 & 16.86 & 0.077 \\
\hline $04-1 \mathrm{~A}$ & 568 & 2.32 & 23.71 & 0.031 & 0.499 & 1.71 & 0.052 & 2.88 & 2.50 & 0.472 & 0.123 & 2.11 & 5.84 & 0.360 \\
\hline $04-2 \mathrm{~A}$ & 317 & 2.60 & 24.82 & 0.021 & 0.359 & 1.24 & 0.031 & 2.27 & 2.28 & 0.654 & 0.114 & 1.49 & 6.05 & 0.251 \\
\hline $04-3 \mathrm{~A}$ & 131 & 2.63 & 26.45 & 0.015 & 0.250 & 0.73 & 0.014 & 1.52 & 1.87 & 0.188 & 0.099 & 0.850 & 14.07 & 0.174 \\
\hline $04-4 \mathrm{~A}$ & 200 & 1.54 & 50.54 & 0.078 & 0.053 & 0.42 & 0.017 & 0.77 & 0.33 & 0.175 & 0.114 & 0.402 & 2.30 & 0.602 \\
\hline $117-1 \mathrm{~A}$ & 51.9 & 2.24 & 14.32 & 0.016 & 0.081 & 0.27 & 0.017 & 1.01 & 0.54 & 0.219 & 0.078 & 0.291 & 1.05 & 0.104 \\
\hline $117-2 \mathrm{~A}$ & 47.3 & 3.90 & 12.33 & 0.006 & 0.061 & 0.22 & 0.008 & 0.76 & 0.38 & 0.069 & 0.060 & 0.249 & 0.74 & 0.061 \\
\hline $117-3 \mathrm{~A}$ & 58.7 & 3.35 & 11.78 & 0.007 & 0.065 & 0.27 & 0.011 & 0.88 & 0.45 & 0.113 & 0.068 & 0.282 & 1.18 & 0.069 \\
\hline $117-4 \mathrm{~A}$ & 80.0 & 1.98 & 13.57 & 0.007 & 0.066 & 0.29 & 0.019 & 1.23 & 0.48 & 0.107 & 0.065 & 0.278 & 25.88 & 0.069 \\
\hline $117-5 \mathrm{~A}$ & 53.7 & 0.963 & 16.51 & 0.008 & 0.097 & 0.29 & 0.025 & 1.04 & 0.36 & 0.098 & 0.073 & 0.320 & 0.977 & 0.083 \\
\hline $117-6 \mathrm{~A}$ & 244 & 0.817 & 10.96 & 0.017 & 0.206 & 0.41 & 0.071 & 1.23 & 0.43 & 0.230 & 0.162 & 0.434 & 19.78 & 0.189 \\
\hline $136-1 \mathrm{~A}$ & 60.2 & 0.424 & 26.09 & 0.022 & 0.055 & 0.15 & 0.066 & 0.52 & 0.38 & 0.151 & 0.069 & 0.174 & 1.14 & 0.079 \\
\hline $136-2 \mathrm{~A}$ & 56.5 & 0.438 & 31.59 & 0.011 & 0.029 & 0.12 & 0.068 & 0.82 & 0.13 & 0.074 & 0.048 & 0.110 & 3.39 & 0.025 \\
\hline $136-3 \mathrm{~A}$ & 26.2 & 0.460 & 33.97 & 0.008 & 0.023 & 0.07 & 0.063 & 0.55 & 0.10 & 0.057 & 0.042 & 0.092 & 0.95 & 0.019 \\
\hline $136-4 \mathrm{~A}$ & 49.0 & 0.400 & 45.67 & 0.013 & 0.024 & 0.13 & 0.130 & 0.62 & 0.28 & 0.089 & 0.040 & 0.061 & 15.79 & 0.020 \\
\hline $136-5 \mathrm{~A}$ & 31.3 & 0.499 & 46.64 & 0.014 & 0.029 & 0.08 & 0.158 & 0.68 & 0.16 & 0.054 & 0.035 & 0.087 & 1.59 & 0.023 \\
\hline $136-6 \mathrm{~A}$ & 24.5 & 0.676 & 15.86 & 0.011 & 0.081 & 0.07 & 0.035 & 0.45 & 0.23 & 0.030 & 0.006 & 0.051 & 1.03 & 0.098 \\
\hline $96-1 \mathrm{~A}$ & 86.5 & 1.61 & 35.52 & 0.020 & 0.122 & 0.30 & 0.032 & 0.85 & 0.40 & 0.144 & 0.051 & 0.372 & 2.12 & 0.132 \\
\hline $96-2 \mathrm{~A}$ & 95.6 & 1.19 & 27.51 & 0.014 & 0.110 & 0.23 & 0.033 & 0.65 & 0.30 & 0.118 & 0.059 & 0.302 & 1.49 & 0.085 \\
\hline $96-3 \mathrm{~A}$ & 86.2 & 1.13 & 29.09 & 0.015 & 0.087 & 0.17 & 0.032 & 0.60 & 0.23 & 0.139 & 0.062 & 0.226 & 1.67 & 0.079 \\
\hline $96-4 \mathrm{~A}$ & 99.5 & 0.810 & 19.65 & 0.013 & 0.086 & 0.15 & 0.040 & 0.52 & 0.18 & 0.150 & 0.077 & 0.204 & 1.31 & 0.057 \\
\hline $96-5 \mathrm{~A}$ & 161 & 0.799 & 13.23 & 0.011 & 0.118 & 0.26 & 0.059 & 1.04 & 0.23 & 0.133 & 0.067 & 0.228 & 9.80 & 0.055 \\
\hline $96-6 \mathrm{~A}$ & 178 & 0.707 & 25.77 & 0.016 & 0.138 & 0.18 & 0.091 & 0.92 & 0.23 & 0.108 & 0.102 & 0.156 & 19.73 & 0.040 \\
\hline
\end{tabular}


Table 4. Cont

\begin{tabular}{|c|c|c|c|c|c|c|c|c|c|c|c|c|c|c|}
\hline Label & Al & As & $\mathbf{B a}$ & $\mathrm{Cd}$ & Co & $\mathrm{Cr}$ & Cs & $\mathrm{Cu}$ & $\mathrm{Ni}$ & $\mathbf{P b}$ & $\mathbf{U}$ & V & $\mathrm{Zn}$ & $\mathbf{L a}$ \\
\hline $97-1 \mathrm{~A}$ & 114 & 1.54 & 12.64 & $<\mathrm{DL}$ & 0.113 & 0.16 & 0.063 & 0.82 & 0.33 & 0.121 & 0.059 & 3.87 & 5.28 & 0.098 \\
\hline $97-2 \mathrm{~A}$ & 176 & 0.662 & 9.15 & $<\mathrm{DL}$ & 0.131 & 0.27 & 0.078 & 1.00 & 0.36 & 0.053 & 0.044 & 1.68 & 17.93 & 0.113 \\
\hline $97-3 \mathrm{~A}$ & 137 & 0.356 & 8.38 & $<\mathrm{DL}$ & 0.110 & 0.26 & 0.048 & 1.08 & 0.34 & 0.065 & 0.042 & 1.65 & 10.29 & 0.094 \\
\hline $97-4 \mathrm{~A}$ & 159 & 0.244 & 6.60 & $<\mathrm{DL}$ & 0.112 & 0.16 & 0.033 & 0.95 & 0.27 & 0.055 & 0.020 & 1.66 & 6.41 & 0.091 \\
\hline $97-5 \mathrm{~A}$ & 201 & 0.234 & 4.46 & $<\mathrm{DL}$ & 0.132 & 0.18 & 0.025 & 0.51 & 0.23 & 0.083 & 0.012 & 2.37 & 0.583 & 0.157 \\
\hline $97-6 \mathrm{~A}$ & 49.4 & 0.247 & 1.02 & $<\mathrm{DL}$ & 0.012 & 0.13 & 0.028 & 0.46 & 0.05 & 0.017 & 0.007 & 3.57 & 6.83 & 0.012 \\
\hline $03-1 \mathrm{~A}$ & 206 & 1.62 & 67.37 & 0.017 & 0.167 & 0.40 & 0.116 & 1.47 & 0.53 & 0.356 & 0.048 & 0.453 & 5.61 & 0.190 \\
\hline $03-2 \mathrm{~A}$ & 140 & 1.61 & 74.90 & 0.037 & 0.124 & 0.25 & 0.113 & 0.83 & 0.33 & 0.295 & 0.028 & 0.258 & 9.28 & 0.097 \\
\hline $03-3 \mathrm{~A}$ & 131 & 1.60 & 50.49 & 0.032 & 0.069 & 0.26 & 0.155 & 0.83 & 0.21 & 0.225 & 0.025 & 0.310 & 2.21 & 0.104 \\
\hline $01-1 \mathrm{~B}$ & 27.8 & 1.40 & 10.04 & 0.015 & 0.059 & 0.17 & 0.042 & 3.12 & 0.35 & 0.170 & 0.234 & 0.324 & 1.10 & 0.184 \\
\hline $01-2 B$ & 19.4 & 0.992 & 10.85 & 0.020 & 0.042 & 0.07 & 0.038 & 2.58 & 0.13 & 0.113 & 0.201 & 0.174 & 1.35 & 0.174 \\
\hline $01-3 B$ & 16.4 & 0.573 & 12.91 & 0.023 & 0.036 & 0.06 & 0.056 & 2.73 & 0.11 & 0.115 & 0.154 & 0.160 & 1.25 & 0.136 \\
\hline 01-4B & 12.8 & 0.648 & 16.84 & 0.014 & 0.059 & 0.11 & 0.027 & 8.00 & 0.22 & 0.145 & 0.200 & 0.173 & 1.03 & 0.100 \\
\hline $02-1 \mathrm{~B}$ & 131 & 2.93 & 116.9 & 0.018 & 0.195 & 0.40 & 0.220 & 1.15 & 0.61 & 0.655 & 0.166 & 1.01 & 4.65 & 0.189 \\
\hline $02-2 B$ & 68.7 & 2.23 & 91.48 & 0.11 & 0.160 & 0.13 & 0.297 & 1.27 & 0.56 & 0.349 & 0.156 & 0.551 & 8.58 & 0.072 \\
\hline $02-3 B$ & 54.1 & 0.999 & 59.26 & 0.016 & 0.101 & 0.13 & 0.443 & 1.64 & 0.22 & 0.202 & 0.155 & 0.441 & 2.18 & 0.049 \\
\hline $58-1 B$ & 26.2 & 0.813 & 28.65 & 0.009 & 0.055 & 0.13 & 0.098 & 6.61 & 0.25 & 0.167 & 0.948 & 0.201 & 1.30 & 0.043 \\
\hline $58-2 B$ & 55.3 & 1.13 & 26.65 & 0.009 & 0.105 & 0.09 & 0.109 & 0.60 & 0.22 & 0.126 & 1.53 & 0.196 & 13.46 & 0.034 \\
\hline $58-3 B$ & 99.3 & 1.87 & 23.35 & 0.011 & 0.144 & 0.13 & 0.273 & 1.05 & 0.26 & 0.448 & 1.23 & 0.417 & 9.72 & 0.063 \\
\hline $58-4 \mathrm{~B}$ & 42.8 & 2.02 & 10.53 & 0.007 & 0.153 & 0.15 & 0.304 & 0.90 & 0.27 & 0.261 & 1.06 & 0.240 & 0.63 & 0.057 \\
\hline $58-5 B$ & 118 & 0.903 & 8.65 & 0.025 & 0.075 & 0.10 & 0.155 & 0.48 & 0.18 & 0.153 & 0.300 & 0.186 & 18.50 & 0.078 \\
\hline $58-6 B$ & 139 & 1.74 & 6.64 & 0.033 & 0.094 & 0.12 & 0.061 & 1.37 & 0.39 & 0.300 & 0.146 & 0.107 & 2.68 & 0.067 \\
\hline $137-1 B$ & 31.6 & 3.09 & 17.61 & 0.022 & 0.107 & 0.22 & 0.126 & 0.72 & 0.84 & 0.176 & 0.931 & 0.463 & 1.04 & 0.126 \\
\hline $137-2 B$ & 36.7 & 2.81 & 26.02 & 0.012 & 0.088 & 0.18 & 0.128 & 0.50 & 0.21 & 0.197 & 0.185 & 0.494 & 0.54 & 0.124 \\
\hline 137-3B & 30.6 & 2.67 & 25.07 & 0.017 & 0.197 & 0.17 & 0.116 & 0.94 & 0.24 & 0.209 & 0.170 & 0.388 & 1.00 & 0.093 \\
\hline $137-4 B$ & 37.2 & 2.23 & 31.77 & 0.027 & 0.123 & 0.22 & 0.120 & 0.78 & 0.22 & 0.207 & 0.164 & 0.375 & 5.99 & 0.096 \\
\hline 137-5B & 34.4 & 2.17 & 21.31 & 0.033 & 0.046 & 0.21 & 0.110 & 0.33 & 0.13 & 0.163 & 0.094 & 0.297 & 7.73 & 0.077 \\
\hline $137-6 B$ & 86.0 & 1.27 & 10.90 & 0.060 & 0.109 & 0.23 & 0.083 & 0.27 & 0.20 & 5.39 & 0.060 & 0.257 & 10.40 & 0.094 \\
\hline 04-1B & 39.9 & 3.09 & 26.93 & 0.013 & 0.107 & 0.31 & 0.009 & 0.78 & 0.83 & 0.147 & 0.062 & 1.19 & 3.49 & 0.104 \\
\hline $04-2 B$ & 24.1 & 3.49 & 27.94 & 0.010 & 0.088 & 0.31 & 0.006 & 0.71 & 0.83 & 0.118 & 0.051 & 1.04 & 0.64 & 0.075 \\
\hline 04-3B & 13.6 & 3.90 & 29.45 & 0.009 & 0.129 & 0.29 & 0.005 & 0.66 & 0.97 & 0.093 & 0.048 & 0.851 & 0.53 & 0.061 \\
\hline $04-4 \mathrm{~B}$ & 20.2 & 3.62 & 41.20 & 0.010 & 0.048 & 0.15 & 0.007 & 0.92 & 0.41 & 0.101 & 0.018 & 0.341 & 2.47 & 0.033 \\
\hline $117-1 B$ & 27.2 & 7.72 & 20.60 & 0.008 & 0.080 & 0.13 & 0.008 & 1.29 & 0.76 & 0.094 & 0.045 & 0.407 & 1.78 & 0.052 \\
\hline $117-2 B$ & 26.2 & 5.73 & 18.05 & 0.009 & 0.062 & 0.16 & 0.008 & 0.79 & 0.45 & 0.114 & 0.060 & 0.317 & 9.97 & 0.067 \\
\hline $117-3 B$ & 53.0 & 5.75 & 17.03 & 0.012 & 0.093 & 0.24 & 0.015 & 1.16 & 0.54 & 0.210 & 0.083 & 0.368 & 2.45 & 0.092 \\
\hline $117-4 B$ & 28.8 & 1.96 & 17.85 & 0.011 & 0.073 & 0.32 & 0.014 & 1.14 & 0.49 & 0.208 & 0.091 & 0.366 & 0.86 & 0.094 \\
\hline 117-5B & 44.3 & 1.82 & 19.27 & 0.011 & 0.099 & 0.29 & 0.025 & 1.75 & 0.42 & 0.274 & 0.084 & 0.400 & 1.51 & 0.099 \\
\hline $117-6 B$ & 270 & 2.66 & 6.82 & 0.017 & 0.679 & 0.38 & 0.020 & 0.35 & 0.52 & 0.696 & 0.174 & 0.709 & 2.09 & 0.218 \\
\hline 136-1B & 14.2 & 0.603 & 30.21 & 0.012 & 0.037 & 0.07 & 0.089 & 0.41 & 0.31 & 0.118 & 0.046 & 0.193 & 1.24 & 0.027 \\
\hline
\end{tabular}


Table 4. Cont.

\begin{tabular}{|c|c|c|c|c|c|c|c|c|c|c|c|c|c|c|}
\hline Label & Al & As & $\mathbf{B a}$ & $\mathrm{Cd}$ & Co & $\mathrm{Cr}$ & Cs & $\mathrm{Cu}$ & $\mathrm{Ni}$ & $\mathrm{Pb}$ & $\mathbf{U}$ & $\mathbf{V}$ & $\mathrm{Zn}$ & La \\
\hline $136-2 B$ & 22.1 & 0.835 & 44.12 & 0.013 & 0.047 & 0.05 & 0.115 & 0.51 & 0.17 & 0.073 & 0.042 & 0.125 & 10.70 & 0.021 \\
\hline 136-3B & 13.6 & 0.788 & 48.10 & 0.013 & 0.036 & $<\mathrm{DL}$ & 0.098 & 0.63 & 0.36 & 0.093 & 0.034 & 0.089 & 3.04 & 0.019 \\
\hline 136-4B & 31.1 & 0.849 & 75.91 & 0.017 & 0.043 & 0.05 & 0.221 & 1.18 & 0.30 & 0.204 & 0.046 & 0.104 & 17.46 & 0.033 \\
\hline $136-5 B$ & 32.4 & 0.756 & 54.97 & 0.013 & 0.049 & 0.05 & 0.196 & 0.66 & 0.24 & 0.149 & 0.039 & 0.105 & 12.24 & 0.029 \\
\hline 136-6B & 36.6 & 0.815 & 16.48 & 0.016 & 0.088 & $<\mathrm{DL}$ & 0.051 & 0.27 & 0.45 & 0.039 & 0.008 & 0.036 & 3.29 & 0.124 \\
\hline $96-1 B$ & 36.3 & 2.64 & 39.74 & 0.013 & 0.070 & 0.18 & 0.034 & 0.46 & 0.27 & 0.142 & 0.042 & 0.395 & 1.10 & 0.072 \\
\hline $96-2 B$ & 40.6 & 2.26 & 30.00 & 0.010 & 0.064 & 0.17 & 0.036 & 0.37 & 0.21 & 0.122 & 0.054 & 0.345 & 0.60 & 0.059 \\
\hline $96-3 B$ & 159 & 2.59 & 32.96 & 0.015 & 0.130 & 0.23 & 0.063 & 0.53 & 0.28 & 0.408 & 0.098 & 0.438 & 3.28 & 0.129 \\
\hline 96-4B & 62.9 & 1.96 & 22.45 & 0.013 & 0.051 & 0.08 & 0.052 & 0.39 & 0.19 & 0.167 & 0.085 & 0.238 & 1.12 & 0.046 \\
\hline 96-5B & 121 & 1.94 & 13.73 & 0.009 & 0.149 & 0.12 & 0.078 & 0.47 & 0.20 & 0.172 & 0.080 & 0.276 & 18.80 & 0.047 \\
\hline $96-6 \mathrm{~B}$ & 5.58 & 3.00 & 4.12 & 0.005 & 0.012 & $<\mathrm{DL}$ & 0.125 & 0.30 & $<\mathrm{DL}$ & 0.015 & 0.336 & 0.048 & 0.33 & 0.001 \\
\hline $97-1 B$ & 69.5 & 3.32 & 15.11 & $<\mathrm{DL}$ & 0.113 & 0.07 & 0.098 & 0.75 & 0.28 & 0.174 & 0.104 & 8.62 & 0.93 & 0.124 \\
\hline $97-2 B$ & 17.15 & 1.90 & 11.63 & $<\mathrm{DL}$ & 0.133 & $<\mathrm{DL}$ & 0.158 & 0.62 & 0.28 & 0.023 & 0.075 & 3.30 & 1.68 & 0.044 \\
\hline $97-3 B$ & 20.22 & 0.679 & 9.98 & $<\mathrm{DL}$ & 0.088 & $<\mathrm{DL}$ & 0.059 & 0.57 & 0.20 & 0.037 & 0.064 & 3.15 & 0.51 & 0.048 \\
\hline $97-4 B$ & 17.98 & 0.374 & 6.59 & $<\mathrm{DL}$ & 0.083 & 0.05 & 0.031 & 0.59 & 0.15 & 0.027 & 0.020 & 2.75 & 0.38 & 0.040 \\
\hline $97-5 B$ & 38.1 & 0.330 & 3.31 & $<\mathrm{DL}$ & 0.083 & $<\mathrm{DL}$ & 0.028 & 0.27 & 0.10 & 0.015 & 0.009 & 3.28 & 0.31 & 0.043 \\
\hline $97-6 B$ & 12.49 & 0.310 & 1.10 & $<\mathrm{DL}$ & 0.022 & 0.07 & 0.026 & 0.05 & 0.02 & 0.014 & 0.008 & 4.11 & 0.25 & 0.015 \\
\hline 03-1B & 23.94 & 4.04 & 91.99 & 0.010 & 0.151 & 0.16 & 0.028 & 3.43 & 0.51 & 0.445 & 0.054 & 0.457 & 1.40 & 0.137 \\
\hline 03-2B & 18.98 & 3.44 & 100.4 & 0.021 & 0.124 & 0.13 & 0.074 & 1.41 & 0.38 & 0.284 & 0.035 & 0.216 & 6.24 & 0.060 \\
\hline $03-3 B$ & 33.8 & 3.25 & 68.67 & 0.034 & 0.077 & 0.13 & 0.148 & 1.88 & 0.26 & 0.208 & 0.023 & 0.271 & 5.81 & 0.087 \\
\hline EQS & $200^{1}$ & $0.83^{2}$ & $60^{2}$ & $<0.08^{3}$ & $0.3^{2}$ & $3.4^{2}$ & & $1^{2}$ & $4^{3}$ & $1.2^{3}$ & $0.3^{2}$ & $2.5^{2}$ & $3.1^{2}$ & \\
\hline
\end{tabular}

(1) Quality standard for drinking waters [53]; (2) Annual average Quality Standard for freshwater ecosystems [53]; (3) Priority substances [54].

Sediments were sampled at the spring, in the stream channel, of each of the 10 selected rivers. About $10 \mathrm{~g}$ of sediments was taken using a plastic shovel and transported in clean plastic bags back to the laboratory. After air drying, sediments were homogenized and then digested by alkaline melting until further analysis for their major and trace element concentrations by the SARM laboratory. Accuracy and precision were evaluated at regular intervals with certified reference materials (CAL-S (carbonate), UB-N (serpentinite), BR (basalt), AN-G (anorthosite), GH (granite), DR-N (diorite)). Data are presented in Table 5. 
Table 5. Chemical analysis of bulk sediments. LOI: loss on ignition. For a given element, extreme values are highlighted (light grey: minimum; rectangle: maximum).

\begin{tabular}{|c|c|c|c|c|c|c|c|c|c|c|c|c|c|}
\hline$\% \mathrm{~g} / \mathrm{g}$ & $\mathrm{SiO}_{2}$ & $\mathrm{Al}_{2} \mathrm{O}_{3}$ & $\mathrm{Fe}_{2} \mathrm{O}_{3}$ & $\mathrm{MnO}$ & $\mathrm{MgO}$ & $\mathrm{CaO}$ & $\mathrm{Na}_{2} \mathrm{O}$ & $\mathrm{K}_{2} \mathrm{O}$ & $\mathrm{TiO}_{2}$ & $\mathrm{P}_{2} \mathrm{O}_{5}$ & LOI & Total & \\
\hline La Durolle-01 & 79.28 & 10.28 & 1.31 & 0.02 & 0.37 & 0.15 & 2.05 & 4.77 & 0.17 & $<\mathrm{DL}$ & 0.91 & 99.30 & \\
\hline Les Echets-02 & 82.46 & 9.27 & 0.61 & $\begin{array}{c}< \\
\mathrm{DL}\end{array}$ & 0.14 & 0.31 & 1.32 & 4.72 & 0.09 & 0.11 & 0.82 & 99.85 & \\
\hline La Couze-58 & 84.95 & 8.26 & 0.79 & 0.02 & 0.13 & 0.09 & 1.01 & 3.03 & 0.10 & 0.12 & 1.80 & 100.30 & \\
\hline Le Chapeauroux-137 & 68.19 & 15.22 & 6.43 & 0.06 & 1.84 & 0.05 & 0.27 & 3.26 & 0.77 & $<\mathrm{DL}$ & 4.24 & 100.31 & \\
\hline La Petite Briance-04 & 81.64 & 9.56 & 1.24 & 0.04 & 0.24 & 0.29 & 1.78 & 3.81 & 0.09 & $<\mathrm{DL}$ & 1.11 & 99.81 & \\
\hline Le Doulon-117 & 51.96 & 12.15 & 10.13 & 0.14 & 6.79 & 8.12 & 2.49 & 2.21 & 2.48 & 0.66 & 2.53 & 99.65 & \\
\hline L'Allier-136 & 75.52 & 10.90 & 2.51 & 0.05 & 1.37 & 1.19 & 1.61 & 4.90 & 0.42 & 0.14 & 1.61 & 100.21 & \\
\hline $\begin{array}{c}\text { La } \\
\text { Combade-96 }\end{array}$ & 81.53 & 9.97 & 0.85 & 0.02 & 0.21 & 0.08 & 0.94 & 4.68 & 0.17 & $<\mathrm{DL}$ & 1.35 & 99.80 & \\
\hline La Sioule-97 & 48.30 & 14.53 & 11.48 & 0.16 & 5.64 & 8.72 & 2.65 & 1.46 & 2.68 & 0.80 & 2.93 & 99.36 & \\
\hline Le Richaufour-03 & 71.30 & 14.04 & 3.77 & 0.08 & 1.27 & 0.23 & 1.32 & 4.43 & 0.53 & 0.11 & 2.85 & 99.92 & \\
\hline$\mu \mathrm{g} / \mathrm{g}$ & As & $\mathbf{B a}$ & $\mathrm{Cd}$ & Co & $\mathrm{Cr}$ & Cs & $\mathrm{Cu}$ & $\mathrm{Ni}$ & $\mathrm{Pb}$ & $\mathbf{U}$ & $\mathbf{V}$ & Zn & La \\
\hline La Durolle-01 & 5.0 & 432 & 0.06 & 1.74 & 13.05 & 4.06 & 2.2 & 4.2 & 31.15 & 2.73 & 10.45 & 24.1 & 12.94 \\
\hline Les Echets-02 & 4.3 & 438 & 0.06 & 1.42 & 6.43 & 11.10 & 3.6 & 2.8 & 47.01 & 2.41 & 6.25 & 29.4 & 13.07 \\
\hline La Couze-58 & 10.5 & 53.6 & 0.02 & 0.44 & 2.71 & 55.45 & 3.4 & $\begin{array}{c}< \\
\mathrm{DL}\end{array}$ & 9.82 & 5.67 & 2.45 & 68.3 & 6.38 \\
\hline Le Chapeauroux-137 & 58.6 & 617 & 0.09 & 9.33 & 71.31 & 7.48 & 26.6 & 31.1 & 17.06 & 3.38 & 85.4 & 73.5 & \\
\hline La Petite Briance-04 & 17.6 & 1025 & 0.06 & 2.56 & 12.02 & 2.05 & 3.8 & 5.2 & 17.40 & 0.95 & 10.49 & 30.3 & \\
\hline Le Doulon-117 & 3.9 & 679 & 0.12 & 37.77 & 221 & 1.80 & 38.1 & 132 & 10.87 & 1.90 & 201 & 95.6 & 47.57 \\
\hline L'Allier-136 & 7.5 & 1210 & 0.24 & 6.57 & 43.24 & 5.16 & 8.2 & 18.2 & 98.2 & 1.72 & 38.71 & 138 & 16.43 \\
\hline $\begin{array}{c}\text { La } \\
\text { Combade-96 }\end{array}$ & 8.1 & 363 & 0.04 & 1.09 & 8.85 & 6.37 & 3.0 & 2.8 & 24.73 & 2.99 & 11.68 & 31.9 & 15.94 \\
\hline La Sioule-97 & 13.9 & 578 & 0.16 & 34.47 & 145.39 & 1.64 & 37.3 & 61 & 6.03 & 1.73 & 249 & 121 & 49.55 \\
\hline Le Richaufour-03 & 37.0 & 1112 & 0.25 & 4.38 & 12.63 & 16.90 & 9.2 & 5.6 & 39.69 & 5.92 & 43.09 & 96.9 & \\
\hline$D L$ & 0.5 & 5.5 & 0.02 & 0.08 & 0.50 & 0.02 & 2.0 & 2.0 & 0.45 & 0.01 & 0.85 & 7.0 & 0.02 \\
\hline
\end{tabular}

\subsubsection{Lead Stable Isotopes}

All water and sediment samples were prepared in a clean laboratory (class-100). After the water samples were centrifuged [55], the Pb isotope ratios $\left({ }^{206} \mathrm{~Pb} /{ }^{204} \mathrm{~Pb},{ }^{207} \mathrm{~Pb} /{ }^{204} \mathrm{~Pb}\right.$, ${ }^{208} \mathrm{~Pb} /{ }^{204} \mathrm{~Pb},{ }^{207} \mathrm{~Pb} /{ }^{206} \mathrm{~Pb}$ and ${ }^{208} \mathrm{~Pb} /{ }^{206} \mathrm{~Pb}$ ) were measured in the supernatant by HR-ICPMS (Attom; Nu Instruments) with an Aridus II desolving membrane as the introduction system. For the sediment samples, $\mathrm{Pb}$ digestion $\left(\mathrm{HF} / \mathrm{HNO}_{3}\right)$ and chemical purification on an anion exchange resin (BioRad AG1X-8, 200-400 mesh) $\mathrm{HBr} / \mathrm{HCl}$ medium followed the procedure described in Manhes et al. [56]. Pb isotope ratios were then measured by MC-ICPMS on a NuPlasma II with an Aridus II desolving membrane as the introduction system. All samples were doped with thallium (Tl) to correct for the mass bias [57]. Measurement of the NBS-981 standard every five samples throughout the analytical sequence allowed for obtaining a $\mathrm{Tl}-\mathrm{Pb}$ mass bias relationship [58]. Measurements of the in-house CGPb1 standard yielded the following long-term averages $(2 \sigma, \mathrm{n}=22$, over several years): ${ }^{208} \mathrm{~Pb} /{ }^{206} \mathrm{~Pb}=2.0425 \pm 0.0003,{ }^{206} \mathrm{~Pb} /{ }^{204} \mathrm{~Pb}=18.596 \pm 0.008,{ }^{207} \mathrm{~Pb} /{ }^{204} \mathrm{~Pb}=15.702 \pm 0.005$, ${ }^{208} \mathrm{~Pb} /{ }^{204} \mathrm{~Pb}=37.98 \pm 0.02$ and ${ }^{207} \mathrm{~Pb} /{ }^{206} \mathrm{~Pb}=0.8444 \pm 0.0002$. For the water samples, errors were: ${ }^{208} \mathrm{~Pb} /{ }^{206} \mathrm{~Pb}=0.003,{ }^{206} \mathrm{~Pb} /{ }^{204} \mathrm{~Pb}=0.057,{ }^{207} \mathrm{~Pb} /{ }^{204} \mathrm{~Pb}=0.050,{ }^{208} \mathrm{~Pb} /{ }^{204} \mathrm{~Pb}=0.07$ and ${ }^{207} \mathrm{~Pb} /{ }^{206} \mathrm{~Pb}=0.0013$. For the sediment samples, errors were: ${ }^{208} \mathrm{~Pb} /{ }^{206} \mathrm{~Pb}=0.0004$, ${ }^{206} \mathrm{~Pb} /{ }^{204} \mathrm{~Pb}=0.006,{ }^{207} \mathrm{~Pb} /{ }^{204} \mathrm{~Pb}=0.005,{ }^{208} \mathrm{~Pb} /{ }^{204} \mathrm{~Pb}=0.014$ and ${ }^{207} \mathrm{~Pb} /{ }^{206} \mathrm{~Pb}=0.0001$. Data are presented in Table S2 (Supplementary Material).

\subsubsection{Reaction Conditions}

Redox reactions play an important role in defining the chemistry of natural waters and drive the mobility of many elements. As redox systems never reach equilibrium in 
natural waters, we did not measure the redox potential of the stream water samples. This happens because several reactions, each one at a distinct rate, can occur simultaneously, resulting in a competition for matter, and they can also be driven by biota $[59,60]$. We elected to use the following 3 parameters to assess the redox conditions of our river waters: (i) dissolved $\mathrm{O}_{2}$ saturation index, (ii) dissolved $\mathrm{Fe}$ concentration and (iii) dissolved $\mathrm{Mn}$ concentration. Redox potential (pe) values were calculated for each stream water using the thermodynamic data provided by the WATEQ4F database [61]. Dissolved Mn was assumed to be solely under the form of the $\mathrm{Mn}^{2+}$ free ion and, accordingly, dissolved iron to be solely $\mathrm{Fe}^{2+}$ free ion, considering that our $\mathrm{pH}$ varied from 5.58 to 7.80 (Table 2). As no $\mathrm{Fe}(\mathrm{OH})_{2}$ can precipitate over this $\mathrm{pH}$ range, we considered that the solid phases were pyrolusite $\left(\mathrm{MnO}_{2}\right)$ and ferrihydrite $\left(\left(\mathrm{Fe}^{3+}\right)_{2} \mathrm{O}_{3}, 0.5 \mathrm{H}_{2} \mathrm{O}\right)$, respectively. Table 2 presents the pe values we calculated for each element (equilibrium between $\mathrm{Fe}^{2+} / \mathrm{Fe}(\mathrm{OH})_{3 a m o r p h o u s}$ and between $\mathrm{Mn}^{2+} / \mathrm{MnO}_{2}$ ). Speciation calculations were made using PHREEQC Version 3.4.0.12927 (2017 version; [62]) with the MINTEQ.V4 database, based on MINTEQA2 [63].

\section{Results}

\subsection{Spring Sediments}

The chemical characteristics of the sediment samples are reported in Table 5. The two samples from the Doulon (gneiss) and the Sioule (basalt) rivers exhibited low $\mathrm{SiO}_{2}$ and rich $\mathrm{Ca}, \mathrm{Mg}, \mathrm{Fe}, \mathrm{Mn}$ and Ti concentrations compared to the other sediments. This is consistent with the basaltic substratum of the Sioule watershed but not with the gneissic nature of the Doulon one, which is mainly composed of gneissic rocks intruded by numerous amphibolite dikes. This suggests that major and trace metal concentrations in the spring sediments mostly reflect the weathering of amphibolitic rocks rather than of gneissic ones.

The granitic and the rhyodacite-andesitic sediments yielded the lowest concentrations for transition metals but were enriched in As and $\mathrm{U}$ when the Doulon and Sioule sediments were rich in transition elements. The Allier sediments exhibited $\mathrm{Ba}, \mathrm{Cd}, \mathrm{Pb}$ and $\mathrm{Zn}$ concentrations that were consistent with the presence of polymetallic sulfide deposits. The Combade sediments exhibited the lowest trace element contents.

\subsection{Reaction Conditions}

Stream waters were lowly mineralized (EC ranged from 17 to $180 \mu \mathrm{S} / \mathrm{cm}$ ) and at near-neutral $\mathrm{pH}$ values (Table 2). As these waters circulated on crystalline rocks, mineral weathering provided mainly cations (except some chloride released from the dissolution of micas and amphiboles). Figure 3 shows that $\mathrm{Ca}^{2+}$ and $\mathrm{Na}^{+}$are the main cations, and $\mathrm{HCO}_{3}{ }^{-}$ is the dominant anion. $\mathrm{HCO}_{3}{ }^{-}$comes from the dissolution of atmospheric $\mathrm{CO}_{2}$, which acts as an electroneutrality buffer. This is consistent with the fact that Ca-bearing minerals are more easily weatherable than Na-bearing ones. Ca is thus usually the dominant cation in young waters circulating over crystalline rocks [64-67]. 


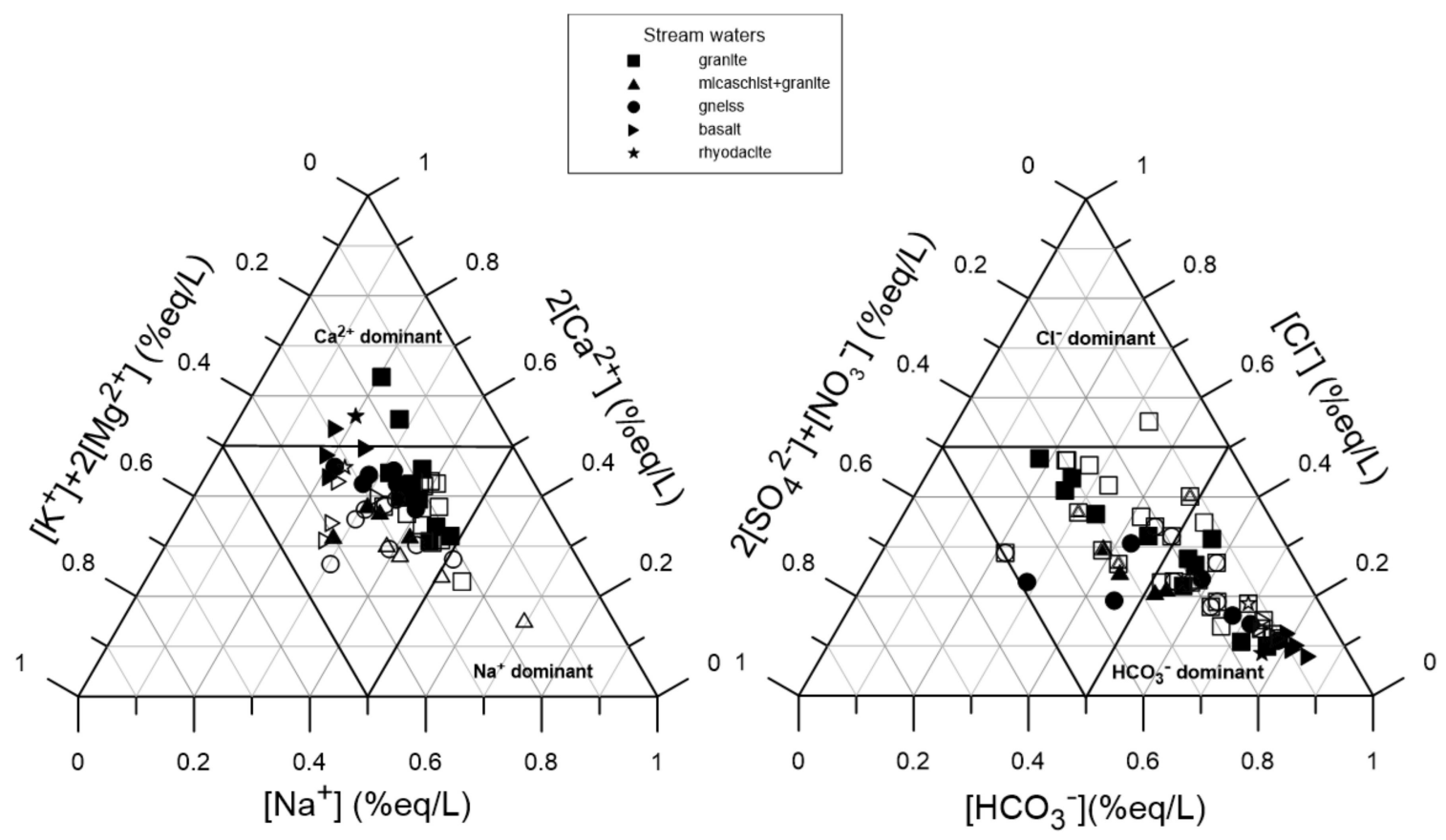

Figure 3. Ion characteristics of the stream water samples according to their lithology. Low-water stage: open symbols; high-water stage: plain symbols.

Results from Figure 3 also show that some rivers were enriched in $\mathrm{Na}^{+}$and $\mathrm{Cl}^{-}$during the low-water stage, which may result from the winter recharge of the aquifers. Based on the study of the major element characteristics, no distinction could clearly be made between the main lithologies of the studied watersheds. Redox conditions recorded by the dissolved oxygen saturation index were distinct between the two sampling seasons (Table 2): from (i) moderately to slightly oxygenated waters during the high-water stage to (ii) oxygenated waters during the low-water stage. The high dissolved Fe (from 5 to $1000 \mu \mathrm{g} \mathrm{L}^{-1}$ ) and dissolved Mn (from 2 to $38 \mu \mathrm{g} \mathrm{L}^{-1}$ ) concentrations suggest that these waters were highly reductive for both seasons, with their reductive character increasing towards the downstream of the rivers. While the pe calculated using these two elements (i.e., Fe and $\mathrm{Mn}$ ) gave distinct values, the respective pe distributions remained similar. The apparent discrepancy between redox conditions recorded by dissolved oxygen and dissolved Fe and Mn can be attributed to different reaction rates: while the dissolved oxygen saturation index records the presence of dissolved oxygen at the time of the measurement, the dissolved Fe and Mn concentrations integrate redox conditions for a longer period of time, according to temperature, $\mathrm{pH}$ and ionic strength [68-70], for kinetics reasons. The redox conditions we measured favored the presence of Fe and $\mathrm{Mn}$ in the dissolved fraction, implying that trace elements usually associated with oxyhydroxide particles were also present in the dissolved fraction.

The DOC concentrations were somewhat identical between the two seasons, with an average value of $3 \pm 2 \mathrm{mgC} \mathrm{L}^{-1}$ (Table 2), but the brownish color of the water samples indicated an important presence of humic substances during the high-water stage.

Under these chemical conditions, the speciation calculations showed that the trace elements presenting different oxidation states were: $\mathrm{As}(+\mathrm{V}), \mathrm{Cr}(+\mathrm{III}), \mathrm{Cu}(+\mathrm{II})$ to $\mathrm{Cu}(+\mathrm{I})$ downstream, $\mathrm{U}(+\mathrm{VI})$ and $\mathrm{V}(+\mathrm{V})$. At the springs, $\mathrm{Cd}, \mathrm{Co}, \mathrm{Cu}, \mathrm{Ni}, \mathrm{Pb}$ and $\mathrm{Zn}$ were mainly present under their free forms, but downstream, the proportion of the complexes they form with $\mathrm{OH}^{-}, \mathrm{CO}_{3}{ }^{2-}$ or $\mathrm{SO}_{4}{ }^{2-}$ increased. Ultimately, our results indicated that both major and minor dissolved elements showed no specific signatures that may have been used to discriminate the different watershed lithologies. This may be explained by the fact that rivers are fed by groundwater, which has minimally circulated through the aquifer, meaning that they underwent a low WRI rate. Thus, water composition is controlled by the 
weatherability of minerals present rather than by the available stock in the host rock. This explains the low differences we observed for the major elements. During the low-water stage, springs were assumed to be representative of the top aquifer.

\subsection{Dissolved Trace Metals and Metalloids}

As expected, most of the dissolved trace element concentrations were below the environmental quality standard (EQS) values, except both As and Zn concentrations, which were above for almost all watersheds (Figure 4). The exceptions (Table 3) were As in (i) the Allier river (WS 136; gneiss; both sampling campaigns), (ii) the upper Sioule (WS 97; basalt; high water stage) and (iii) the Couze (WS 58; granite; high water stage), and Zn in the Sioule river at the low-water stage. The highest $\mathrm{Ba}$ concentrations were observed in the Echets river (WS 02; outlet: granite) but were not correlated to the chemical composition of the spring sediments (Table 4) nor to the presence of a Ba ore deposit. The Sioule River (WS 97 , basalt) exhibited the lowest trace element concentrations except for V. For all monitored rivers, $\mathrm{Cr}$ and $\mathrm{Ni}$ concentrations were significantly below their respective EQS values.

Even if granitic watersheds were richer in $\mathrm{Pb}$ (e.g., the Chapeauroux spring, WS 137) and in $U$ (e.g., the Couze station \#3), no significant correlation could be made between the main lithology and the trace element concentrations. These two watersheds comprise U-rich mineralization deposits (Table 1).

Most studies dealing with the mobility of trace elements based on sequential extractions concluded with their long-time storage in soils and thus attributed a minor importance to leaching losses [71]. Dynamic studies on drainage water assessed important inputs of trace elements from topsoils to the river during rain events [72,73]. Comparing elemental concentrations between the high- and low-water stages and using their differences, expressed as [high-water stage concentration]-[low-water stage concentration], allowed us to better constrain the origin of TMM (Table 6): A negative value indicated a dilution effect occurring during the high-water stage, whereas a positive one indicated an important input of the considered element during the high-water stage, which may result from topsoil leaching and/or atmospheric deposition. Values inferior to the corresponding analytical error were not considered in the calculation.

For all watersheds, while As, Ba and $\mathrm{V}$ were not brought by running waters, $\mathrm{Al}$ and, to a lesser extent, La were (Table 6). Cd concentrations displayed no seasonal contrasts, and the corresponding background levels seemed to depend on the considered watershed but not on the hydrologic conditions (Table 4). Higher Cd concentrations were generally measured at the spring of each river, except for the Durolle River (WS 01, which was expected to be impacted by local road traffic). The Allier River (WS 136) exhibited the smallest seasonal variations. In areas where agricultural activities were more pronounced, this translated into higher $\mathrm{Cr}, \mathrm{Cu}, \mathrm{Ni}, \mathrm{Pb}, \mathrm{U}$ and $\mathrm{Zn}$ (WS 02, 04, 97 and 03) inputs in the running waters. The same trend was observed for $\mathrm{Al}$ and $\mathrm{La}$.

Except for As, our results were consistent with those reported in the literature for other watersheds from the Loire Basin (Figure 4; Table 7; geographic coordinates are given in Table S3 in Supplementary Material). Similar to our results, previous studies concluded that differences in concentrations between the main lithologies were not greater than those recorded within a single lithology facies. This confirms that the chemistry of the watershed depends on local trace element signatures. 

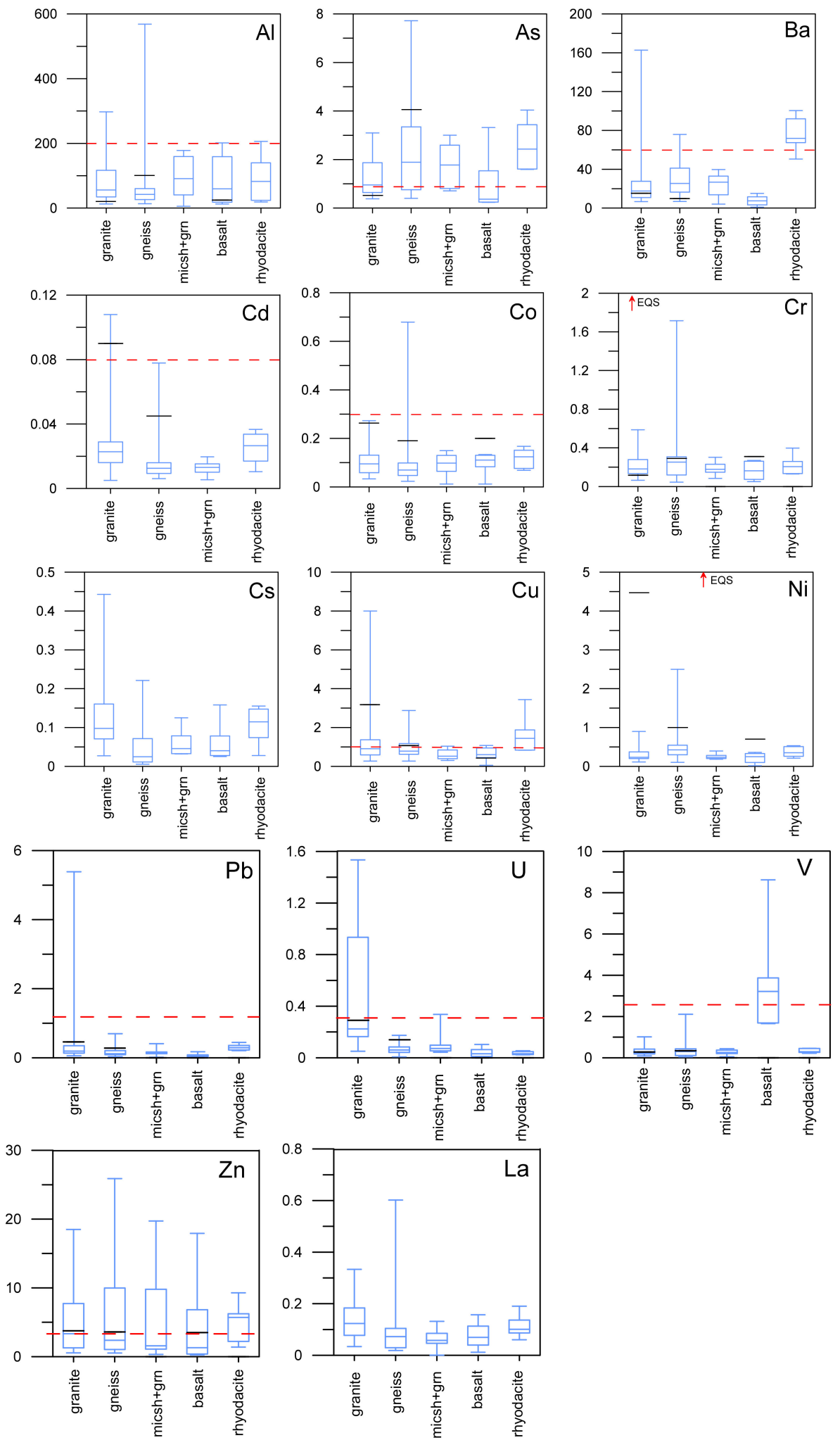

Figure 4. Boxplots showing the concentration distributions for trace elements in the stream water samples according to the main lithology of the corresponding watershed. Concentrations are expressed in $\mu \mathrm{g} \mathrm{L}^{-1}$. The dashed lines mark the environmental quality standard (EQS) values for each element. No EQS is available for Cs and La. For granitic, gneissic and basaltic watersheds, the black dash marks the maximum concentration measured in the collected data, for available elements. 
Table 6. Difference between trace element concentrations between the high- (grey) and low-water stages (expressed as $\left.\mu \mathrm{g} \mathrm{L}^{-1}\right)$. The difference equals the concentration measured during the high-water stage minus the one measured during the low one. AE: analytical error.

\begin{tabular}{|c|c|c|c|c|c|c|c|c|c|c|c|c|c|c|c|}
\hline Lithology & Label & Al & As & Ba & $\mathrm{Cd}$ & Co & $\mathrm{Cr}$ & Cs & $\mathrm{Cu}$ & $\mathrm{Ni}$ & $\mathrm{Pb}$ & $\mathbf{U}$ & $\mathbf{V}$ & Zn & La \\
\hline \multirow{18}{*}{ granite } & $01-1$ & 3 & -0.75 & 0.80 & 0.02 & $<\mathrm{AE}$ & 0.28 & $<\mathrm{AE}$ & -2.13 & 0.21 & 0.07 & 0.02 & -0.13 & 1.20 & 0.036 \\
\hline & $01-2$ & 47 & -0.45 & -0.79 & $<\mathrm{AE}$ & $<\mathrm{AE}$ & 0.09 & 0.00 & -1.72 & $<\mathrm{AE}$ & 0.01 & 0.02 & $<\mathrm{AE}$ & 4.78 & 0.014 \\
\hline & $01-3$ & 30 & -0.19 & $<\mathrm{AE}$ & $<\mathrm{AE}$ & $<\mathrm{AE}$ & $<\mathrm{AE}$ & -0.01 & -2.13 & $<\mathrm{AE}$ & 0.02 & 0.03 & $<\mathrm{AE}$ & $<\mathrm{AE}$ & 0.048 \\
\hline & $01-4$ & 13 & -0.16 & -2.01 & $<\mathrm{AE}$ & 0.07 & $<\mathrm{AE}$ & 0.01 & -7.21 & 0.03 & 0.05 & -0.13 & $<\mathrm{AE}$ & 1.12 & 0.087 \\
\hline & $02-1$ & 167 & -1.58 & 45.75 & $<\mathrm{AE}$ & 0.08 & 0.19 & 0.03 & 0.33 & 0.29 & -0.02 & 0.01 & -0.15 & 0.78 & 0.041 \\
\hline & $02-3$ & 120 & -0.28 & 56.97 & 0.02 & 0.03 & 0.20 & 0.00 & -0.53 & 0.15 & 0.17 & 0.02 & $<\mathrm{AE}$ & 4.95 & 0.037 \\
\hline & $58-1$ & 46 & -0.24 & 2.68 & 0.02 & 0.07 & $<\mathrm{AE}$ & -0.02 & -5.38 & 0.22 & 0.20 & -0.01 & $<\mathrm{AE}$ & 2.05 & 0.071 \\
\hline & $58-2$ & -20 & -0.74 & -2.03 & $<\mathrm{AE}$ & -0.07 & 0.09 & -0.03 & -0.04 & $<\mathrm{AE}$ & -0.07 & -0.32 & -0.11 & -10.8 & 0.109 \\
\hline & $58-3$ & 79 & -1.23 & -2.68 & 0.02 & 0.02 & 0.15 & -0.14 & -0.03 & 0.04 & -0.26 & 0.11 & -0.20 & -2.47 & 0.271 \\
\hline & $58-4$ & 58 & -1.54 & -0.83 & 0.02 & -0.06 & $<\mathrm{AE}$ & -0.15 & -0.40 & 0.05 & -0.04 & 0.22 & -0.10 & 0.93 & 0.039 \\
\hline & $58-5$ & 81 & -0.31 & -1.29 & $<\mathrm{AE}$ & 0.05 & 0.12 & 0.01 & 0.38 & 0.05 & 0.10 & 0.03 & 0.09 & -13.9 & 0.063 \\
\hline & $58-6$ & 106 & -0.92 & 1.02 & $<\mathrm{AE}$ & 0.05 & 0.19 & 0.12 & 0.20 & -0.14 & 0.08 & 0.27 & 0.38 & 3.85 & 0.143 \\
\hline & $137-1$ & 78 & -2.02 & -0.73 & $<\mathrm{AE}$ & $<\mathrm{AE}$ & 0.13 & -0.05 & 0.44 & -0.04 & -0.04 & 0.21 & -0.11 & 7.06 & 0.048 \\
\hline & $137-2$ & 19 & -1.87 & -1.38 & $<\mathrm{AE}$ & -0.02 & 0.09 & -0.05 & 0.09 & $<\mathrm{AE}$ & -0.10 & 0.05 & -0.19 & 0.23 & 0.022 \\
\hline & $137-3$ & 22 & -1.72 & -1.91 & $<\mathrm{AE}$ & -0.12 & 0.11 & -0.05 & -0.54 & $<\mathrm{AE}$ & -0.09 & 0.07 & -0.11 & $<\mathrm{AE}$ & 0.049 \\
\hline & $137-4$ & 79 & -1.34 & -4.14 & $<\mathrm{AE}$ & -0.03 & 0.09 & -0.04 & 0.13 & $<\mathrm{AE}$ & -0.07 & 0.09 & -0.08 & 3.92 & 0.042 \\
\hline & $137-5$ & 57 & -1.20 & -5.05 & $<\mathrm{AE}$ & $<\mathrm{AE}$ & 0.17 & -0.03 & 0.16 & 0.03 & 0.01 & 0.01 & -0.04 & -2.29 & 0.026 \\
\hline & $137-6$ & 21 & -0.65 & -1.96 & $<\mathrm{AE}$ & -0.06 & $<\mathrm{AE}$ & 0.01 & 0.19 & -0.05 & -2.75 & -0.01 & $<\mathrm{AE}$ & 6.46 & -0.017 \\
\hline \multirow{16}{*}{ gneiss } & 04-1 & 528 & -0.77 & -3.23 & 0.02 & 0.39 & 1.41 & 0.04 & 2.10 & 1.67 & 0.33 & 0.06 & 0.92 & 2.35 & 0.256 \\
\hline & 04-2 & 293 & -0.88 & -3.12 & $<\mathrm{AE}$ & 0.27 & 0.94 & 0.02 & 1.56 & 1.45 & 0.54 & 0.06 & 0.45 & 5.41 & 0.176 \\
\hline & 04-3 & 117 & -1.27 & -2.99 & $<\mathrm{AE}$ & 0.12 & 0.44 & 0.01 & 0.86 & 0.90 & 0.10 & 0.05 & $<\mathrm{AE}$ & 13.54 & 0.113 \\
\hline & 04-4 & 180 & -2.08 & 9.34 & 0.07 & $<\mathrm{AE}$ & 0.26 & 0.01 & -0.15 & -0.08 & 0.07 & 0.10 & 0.06 & -0.17 & 0.569 \\
\hline & $117-1$ & 25 & -5.48 & -6.28 & $<\mathrm{AE}$ & $<\mathrm{AE}$ & 0.13 & 0.01 & -0.28 & -0.23 & 0.13 & 0.03 & -0.12 & -0.73 & 0.053 \\
\hline & $117-2$ & 21 & -1.83 & -5.72 & $<\mathrm{AE}$ & $<\mathrm{AE}$ & $<\mathrm{AE}$ & $<\mathrm{AE}$ & -0.03 & -0.07 & -0.05 & $<\mathrm{AE}$ & -0.07 & -9.23 & -0.006 \\
\hline & $117-3$ & 6 & -2.40 & -5.25 & $<\mathrm{AE}$ & 0.03 & $<\mathrm{AE}$ & 0.00 & -0.27 & -0.10 & -0.10 & -0.01 & -0.09 & -1.27 & -0.023 \\
\hline & $117-4$ & 51 & $<\mathrm{AE}$ & -4.29 & $<\mathrm{AE}$ & $<\mathrm{AE}$ & $<\mathrm{AE}$ & 0.01 & 0.09 & $<\mathrm{AE}$ & -0.10 & -0.03 & -0.09 & 25.02 & -0.025 \\
\hline & $117-5$ & 9 & -0.86 & -2.76 & $<\mathrm{AE}$ & $<\mathrm{AE}$ & $<\mathrm{AE}$ & $<\mathrm{AE}$ & -0.71 & -0.06 & -0.18 & -0.01 & -0.08 & -0.53 & -0.016 \\
\hline & $117-6$ & -26 & -1.84 & 4.13 & $<\mathrm{AE}$ & -0.47 & $<\mathrm{AE}$ & 0.05 & 0.88 & -0.09 & -0.47 & -0.01 & -0.28 & 17.70 & -0.028 \\
\hline & $136-1$ & 46 & -0.18 & -4.12 & $<\mathrm{AE}$ & 0.02 & $<\mathrm{AE}$ & -0.02 & 0.11 & 0.08 & 0.03 & 0.02 & $<\mathrm{AE}$ & -0.10 & 0.052 \\
\hline & $136-2$ & 34 & -0.40 & -12.53 & $<\mathrm{AE}$ & -0.02 & $<\mathrm{AE}$ & -0.05 & 0.31 & -0.04 & $<\mathrm{AE}$ & 0.01 & $<\mathrm{AE}$ & -7.31 & 0.004 \\
\hline & $136-3$ & 13 & -0.33 & -14.13 & $<\mathrm{AE}$ & $<\mathrm{AE}$ & $<\mathrm{AE}$ & -0.04 & -0.08 & -0.26 & -0.04 & 0.01 & $<\mathrm{AE}$ & -2.09 & -0.001 \\
\hline & $136-4$ & 18 & -0.45 & -30.23 & $<\mathrm{AE}$ & -0.02 & $<\mathrm{AE}$ & -0.09 & -0.56 & $<\mathrm{AE}$ & -0.11 & -0.01 & -0.04 & -1.68 & -0.014 \\
\hline & $136-5$ & $<\mathrm{AE}$ & -0.26 & -8.33 & $<\mathrm{AE}$ & -0.02 & $<\mathrm{AE}$ & -0.04 & $<\mathrm{AE}$ & -0.08 & -0.10 & 0.00 & $<\mathrm{AE}$ & -10.65 & -0.007 \\
\hline & $136-6$ & -12 & -0.14 & -0.62 & $<\mathrm{AE}$ & $<\mathrm{AE}$ & $<\mathrm{AE}$ & -0.02 & 0.17 & -0.23 & -0.01 & $<\mathrm{AE}$ & $<\mathrm{AE}$ & -2.26 & -0.026 \\
\hline \multirow{6}{*}{ micaschist+granite } & $96-1$ & 50 & -1.04 & -4.22 & $<\mathrm{AE}$ & 0.05 & 0.12 & $<\mathrm{AE}$ & 0.38 & 0.13 & $<\mathrm{AE}$ & 0.01 & $<\mathrm{AE}$ & 1.02 & 0.060 \\
\hline & $96-2$ & 55 & -1.07 & -2.49 & $<\mathrm{AE}$ & 0.05 & $<\mathrm{AE}$ & 0.00 & 0.27 & 0.09 & $<\mathrm{AE}$ & 0.00 & -0.04 & 0.89 & 0.027 \\
\hline & $96-3$ & -73 & -1.46 & -3.87 & $<\mathrm{AE}$ & -0.04 & $<\mathrm{AE}$ & -0.03 & 0.08 & -0.05 & -0.27 & -0.04 & -0.21 & -1.60 & -0.050 \\
\hline & $96-4$ & 37 & -1.15 & -2.80 & $<\mathrm{AE}$ & 0.03 & $<\mathrm{AE}$ & -0.01 & 0.13 & $<\mathrm{AE}$ & -0.02 & -0.01 & $<\mathrm{AE}$ & 0.18 & 0.010 \\
\hline & $96-5$ & 41 & -1.14 & -0.50 & $<\mathrm{AE}$ & -0.03 & 0.14 & -0.02 & 0.57 & 0.03 & -0.04 & -0.01 & -0.05 & -9.00 & 0.008 \\
\hline & $96-6$ & 172 & -2.30 & 21.65 & $<\mathrm{AE}$ & 0.13 & 0.18 & -0.03 & 0.61 & 0.23 & 0.09 & -0.23 & 0.11 & 19.40 & 0.039 \\
\hline \multirow{6}{*}{ basalt } & $97-1$ & 44 & -1.79 & -2.47 & $<\mathrm{AE}$ & $<\mathrm{AE}$ & 0.09 & -0.04 & 0.07 & 0.05 & -0.05 & -0.05 & -4.76 & 4.35 & -0.025 \\
\hline & $97-2$ & 159 & -1.23 & -2.48 & $<\mathrm{AE}$ & $<\mathrm{AE}$ & 0.27 & -0.08 & 0.38 & 0.08 & 0.03 & -0.03 & -1.62 & 16.24 & 0.069 \\
\hline & $97-3$ & 117 & -0.32 & -1.59 & $<\mathrm{AE}$ & 0.02 & 0.26 & -0.01 & 0.50 & 0.14 & 0.03 & -0.02 & -1.50 & 9.78 & 0.046 \\
\hline & $97-4$ & 141 & -0.13 & $<\mathrm{AE}$ & $<\mathrm{AE}$ & 0.03 & 0.11 & $<\mathrm{AE}$ & 0.36 & 0.12 & 0.03 & $<\mathrm{AE}$ & -1.09 & 6.03 & 0.052 \\
\hline & $97-5$ & 163 & -0.10 & 1.15 & $<\mathrm{AE}$ & 0.05 & 0.18 & 0.00 & 0.24 & 0.13 & 0.07 & 0.00 & -0.92 & 0.27 & 0.114 \\
\hline & $97-6$ & 37 & -0.06 & -0.08 & $<\mathrm{AE}$ & $<\mathrm{AE}$ & $<\mathrm{AE}$ & $<\mathrm{AE}$ & 0.40 & 0.03 & $<\mathrm{AE}$ & $<\mathrm{AE}$ & -0.54 & 6.58 & -0.003 \\
\hline \multirow{3}{*}{ rhyodacite } & 03-1 & 182 & -2.42 & -24.62 & $<\mathrm{AE}$ & 0.02 & 0.23 & 0.09 & -1.96 & 0.03 & -0.09 & -0.01 & $<\mathrm{AE}$ & 4.21 & 0.053 \\
\hline & 03-2 & 121 & -1.83 & -25.4 & 0.02 & $<\mathrm{AE}$ & 0.11 & 0.04 & -0.58 & -0.05 & 0.01 & -0.01 & 0.04 & 3.04 & 0.037 \\
\hline & 03-3 & 97 & -1.65 & -18.17 & $<\mathrm{AE}$ & $<\mathrm{AE}$ & 0.13 & 0.01 & -1.05 & -0.05 & 0.02 & $<\mathrm{AE}$ & 0.04 & -3.60 & 0.018 \\
\hline
\end{tabular}


Table 7. Data from the literature. Concentrations are expressed in $\mu \mathrm{g} \mathrm{L}^{-1}$. Text in bold italic corresponds to considered river region.

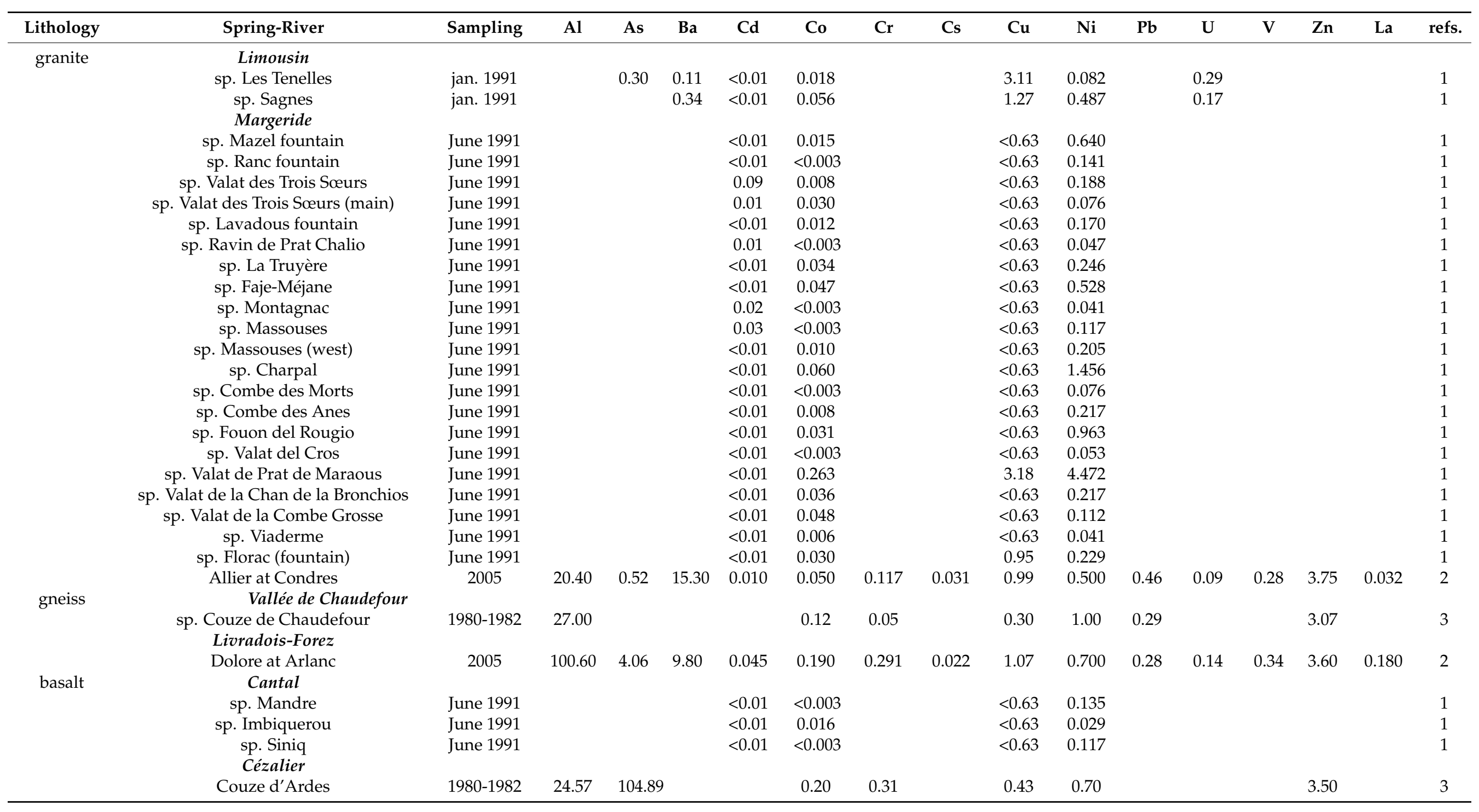


Table 7. Cont.

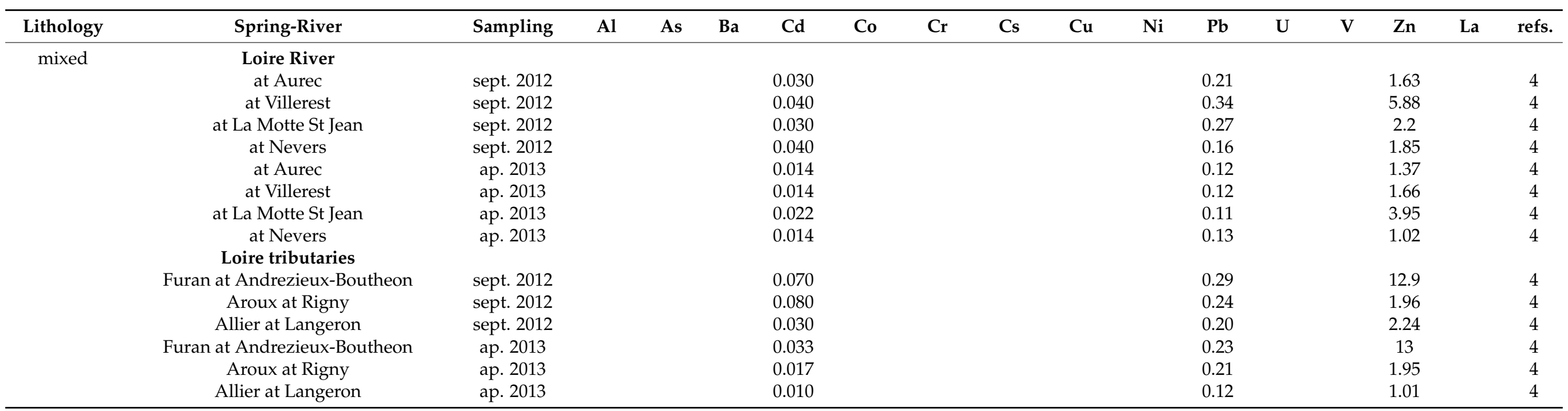

(1) Gassama, [45]; (2) Salminen et al., [13]; (3) Criaud, [44]; (4) stations are sorted out up to downstream, Millot and Desaulty, [46]. 
The ISOP study site, located downstream in the Upper Loire Basin and draining a larger surface area, showed a dilution effect by running waters for $\mathrm{Cd}$ and $\mathrm{Pb}$ (i.e., September: low waters; April: high waters), in contradiction with our results.

For the Allier River watershed, we used published data from one FOREGS (the Allier River at Condres) and one ISOP (the Allier River at Langeron) station to complement our dataset (Figure 1). Results (Figure 5) show that $\mathrm{Cr}, \mathrm{Cs}, \mathrm{Pb}, \mathrm{U}$ and $\mathrm{V}$ follow two distinct trends upstream and downstream of station \#2. No particular trend was observed for Cd. Downstream station \#2, the important volume of water coming from the tributaries smooths the Allier headwater chemical signature. This results in the need to use distinct geochemical baseline values according to the distance from the spring.
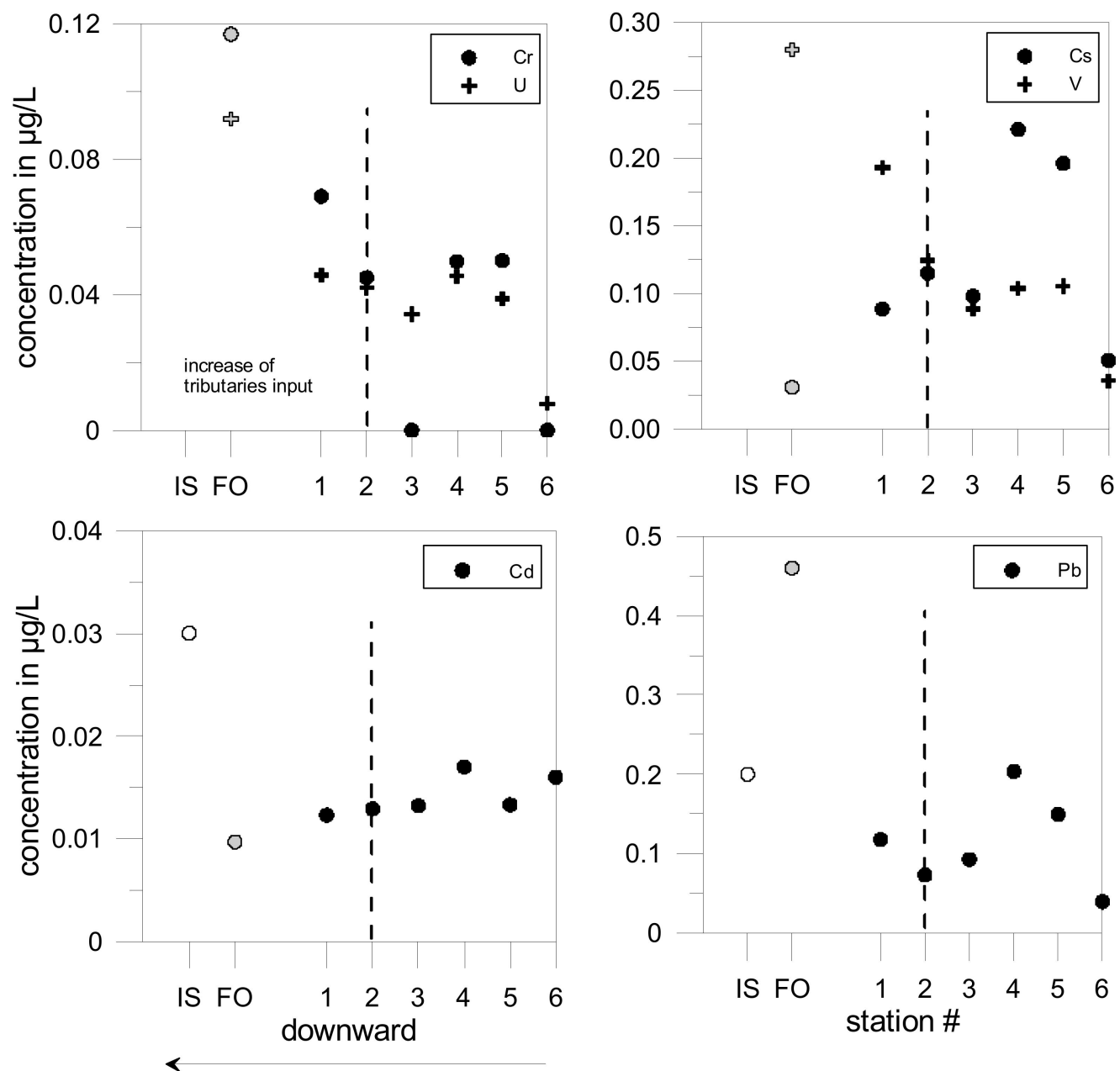

Figure 5. The Allier watershed. Black symbols: this study for low-water stage; grey: FOREGS; empty: ISOP.

\subsection{Lead Stable Isotopes}

Figure 6a reports the lead isotope ratio ranges we measured for the main lithologies of the studied watersheds: granite, quartzitic diorite, rhyolite, gneiss, micaschist and amphibolite. Results show that no clear isotope discrimination can be made according to the nature of the rocks. In stream waters, $\mathrm{Pb}$ can originate from WRI, topsoil leaching and atmospheric deposition. Due to its low mobility in most soils [7,71,74], Pb deposited from the atmosphere and emitted by agricultural activities accumulates in topsoils. For a given area, this can imprint the topsoil with a $\mathrm{Pb}$ isotope signature that is distinct from the geogenic one [75]. Rainwater data in Figure 6a are taken from Roy and Négrel [76] and correspond to samples collected in the nearby city of Clermont-Ferrand (Massif Central, 
France). Négrel et al. [77] chemically characterized rainwater for the same region and showed that while the order of cation abundance was $\mathrm{Ca}^{2+}>\mathrm{Na}^{+}>\mathrm{K}^{+}>\mathrm{Mg}^{2+}$, the order of anion abundance was $\mathrm{NO}_{3}{ }^{-}>\mathrm{SO}_{4}{ }^{2-}>\mathrm{Cl}^{-}$. The $\mathrm{Ca}^{2+}$ and $\mathrm{Na}^{+}$species typically gave mean weighted concentrations of 18 and $14 \mu \mathrm{mol} \mathrm{L}-1$, whereas those for $\mathrm{NO}_{3}{ }^{-}, \mathrm{SO}_{4}{ }^{2-}$ and $\mathrm{Cl}^{-}$were very similar $\left(26,23\right.$ and $19 \mu \mathrm{mol} \mathrm{L}^{-1}$, respectively). To our knowledge, only few $\mathrm{Pb}$ isotope ratios have been published on fertilizers and manure. Still, we are reporting data here corresponding to several types of phosphate fertilizers (nitrogenphosphate, tripplesuperphosphate and diammoniumphosphate [74]), NPK fertilizers (from the ISOP program; nitrogen-phosphate-potassium: N-P-K 17-15-15) and manure (dairy cattle, pig and poultry). $\mathrm{Pb}$ isotope ratios for leaded gasoline were taken from Véron et al. [78] and Widory et al. [79], as they represent the typical ranges for gasoline sold in France and agree with the ranges recently published by Resongles et al. [80]. Results show that fertilizer and manure samples display radiogenic ratios (i.e., high $\mathrm{Pb}$ isotope ratios) comparable to the highest isotope ratios we measured for the different lithologies (Figure 6a) and in the same range as that of regional rainwater [76]. Gasoline is characterized by the most radiogenic values $\left({ }^{208} \mathrm{~Pb} /{ }^{206} \mathrm{~Pb}>\sim 2.12\right.$ and $\left.{ }^{207} \mathrm{~Pb} /{ }^{206} \mathrm{~Pb}>\sim 0.885\right)$, higher than the ones measured in our water and sediment samples. This suggests that road traffic did not significantly contribute to the $\mathrm{Pb}$ budget of the studied stream waters as well as to their adjacent topsoils. Water and sediments mostly yielded $\mathrm{Pb}$ isotope ratios similar to those we measured/reported for rainwater, fertilizer/manure and lithologies (Figure 6b). The exceptions were some water (the Couze, the Chapeauroux; granite lithology) and sediment (the Couze, the Allier, the Combade, the Sioule, the Echets; mixed lithologies) samples that have lower ${ }^{208} \mathrm{~Pb} /{ }^{206} \mathrm{~Pb}$ and ${ }^{207} \mathrm{~Pb} /{ }^{206} \mathrm{~Pb}$ ratios. The three watersheds suspected to be impacted by agriculture practices (the Echets, the Petite Briance, the Richaufour) do not exhibit particular isotope signatures. For all sampling locations, $\mathrm{Pb}$ isotope ratios are lower at the low-water stage than at the high-water stage, suggesting that running waters are characterized by more radiogenic ratios, except for the Sioule and the Petite Briance watersheds that exhibit higher $\mathrm{Pb}$ ratios at the low-water stage. Isotope ratios generally decrease with distance from the spring before increasing at the outlet, as exemplified in Figure $6 \mathrm{c}$ For all watersheds, except the Petite Briance and the Chapeauroux, stream waters show higher isotope ratios compared to the spring sediments (Figure 6c). For the Petite Briance and the Chapeauroux, spring water and spring sediments have similar $\mathrm{Pb}$ isotope ratios, which are higher than those measured in the corresponding water samples collected downstream. If we consider that the spring sediments are not impacted by $\mathrm{Pb}$ coming from topsoil leaching, then $\mathrm{Pb}$ can be considered as solely controlled by the water-rock interaction (WRI) for these two watersheds. For the other streams, the upper watersheds seem impacted by agricultural activities (mainly pasture rearing), as we concluded that road traffic does not contribute, that generate higher $\mathrm{Pb}$ isotope ratios. The isotope ratios then decrease downstream when the streams circulate in more forested areas (Figure 2). As the aggregated drained area increases along the stream, the $\mathrm{Pb}$ flux resulting from topsoil leaching becomes more important. It follows that at the outlets, the topsoil signature overprints the geogenic one. For the Allier watershed, the dilution of the $\mathrm{Pb}$ concentrations recorded at station \#3 is well correlated to the decrease in the $\mathrm{Pb}$ isotope ratios observed at downstream stations (Figure 5). 

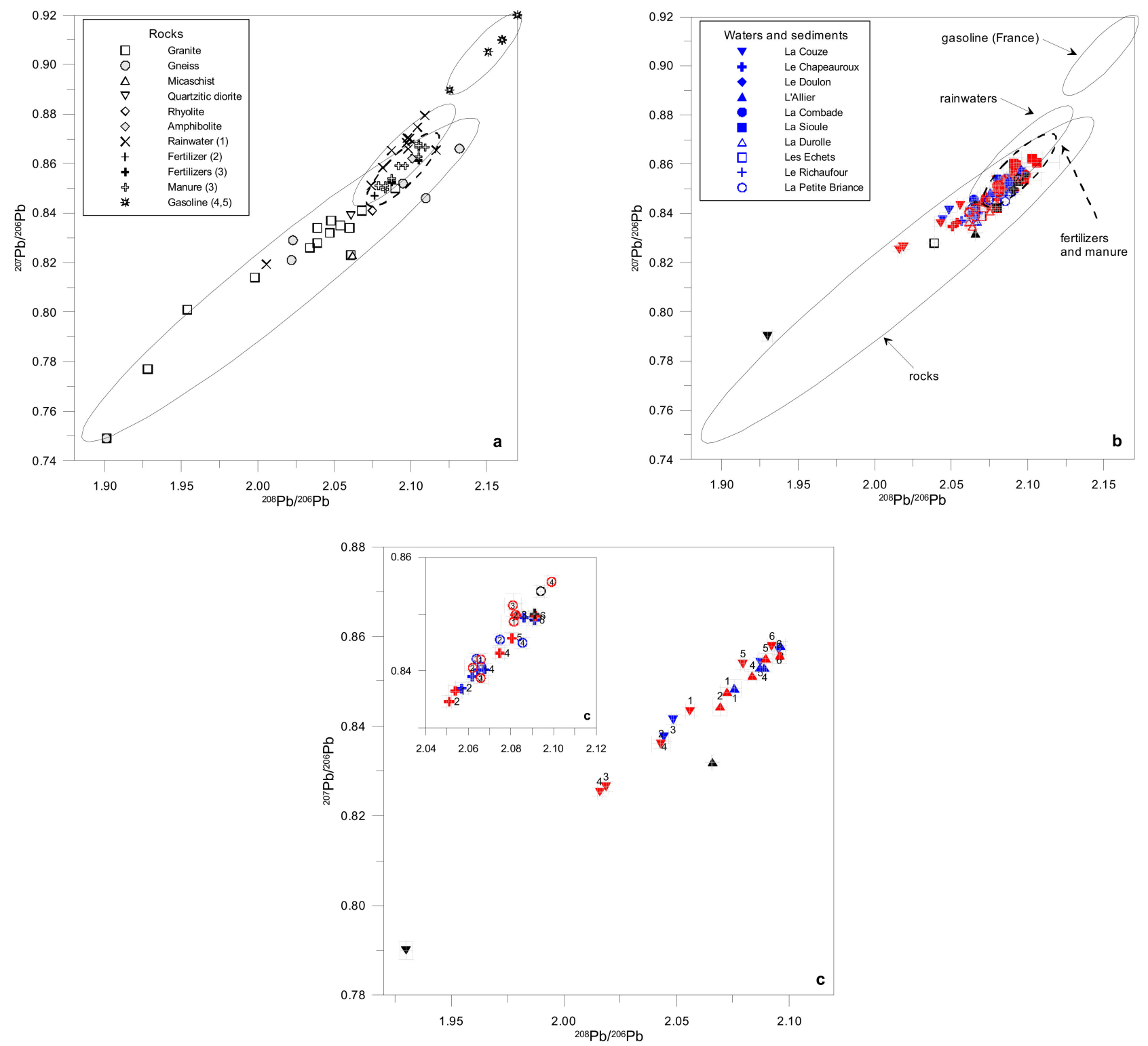

Figure 6. Lead stable isotopes. Plots of ${ }^{207} \mathrm{~Pb} /{ }^{206} \mathrm{~Pb}$ versus ${ }^{208} \mathrm{~Pb} /{ }^{206} \mathrm{~Pb}$ (a) in bulk rocks, fertilizers, manure and leaded gasoline; (b) in waters and sediments; (c) focused on the three watersheds of the Couze, the Allier and the Chapeauroux. Blue: high-water stage; red: low-water stage; black: sediments; grey: standard deviation. (1) Roy and Négrel [76]; (2) Walraven et al. [74]; (3) Millot and Desaulty [45]; (4) Véron et al. [78]; (5) Widory et al. [79].

\section{Discussion}

Our results show that the presence and abundances of TMM in the stream waters of the Upper Loire Basin are not uniquely controlled by WRI. No upstream/downstream trend or direct correlation with the redox conditions was observed. The difference in the TMM concentrations between the high- and low-water stages clearly indicates inputs from running waters (i.e., soil leaching + atmospheric deposition) and/or dilution by meteoric waters. At the low-water stage, the quality of stream waters is driven by the water-rock interaction process and by land cover and atmospheric inputs. If we assume that the chemistry of each water sample collected at the spring is, during this period, solely derived from that of the underlying aquifer, our results demonstrate that most of the watersheds we selected, even if their agriculture practices were $<50 \%$, are likely impacted by agricultural practices for some of the TMM. 


\subsection{Origin of TMM in Stream Waters}

In crystalline areas, watersheds $>20-50 \mathrm{~km}^{2}$ cannot be considered as truly monolithologic, as granitic rocks occur as massive bodies associated with acid and basic rock dikes [81]. Moreover, the distribution of trace metals can highly vary within a granite body and between granites [82-84]. In addition, basic rocks contain more trace metal-bearing minerals, which are more easily weatherable compared to granite minerals (feldspar and quartz). The same features can be observed in gneissic or micaschist areas, but fracturing adds an additional layer of heterogeneity. The relationship existing between the main fracturing direction and runoff is also critical: the more the runoff is perpendicular to the fracturing, the more the waters are mixed and exhibit a homogeneous chemical composition. If plutonic and metamorphic rocks occur as massive bodies, volcanic rocks of several tenths to hundreds of meters in thickness can be found in the basin. Waters may thus circulate not only within lava rocks, even if their permeability favors water circulation, but also within granitic or gneissic rocks (basement), ultimately influencing their chemistry.

Superficial aquifers are encountered at small dimensions in crystalline areas: they have developed in the rock fracturing and in weathering product lenses (i.e., alterites). Although the water-rock interaction is low, the chemical composition of water circulating in these aquifers is controlled by the more easily weatherable minerals, which may not correspond to the most abundant ones. Thus, relationships may not be established between the main lithology (i.e., monolithology) and the geochemical background of the considered watershed. In our study, only $\mathrm{Pb}$ concentrations can be directly linked to the granite lithology, independently of the water stage. The same conclusion can be drawn for $U$, but only when WRI controls the water chemistry, i.e., during the low-water stage.

Trace elements in water can originate from four distinct sources: (i) water-rock interaction, (ii) soil leaching (for which solubility can be enhanced through the formation of organic complexes), (iii) fertilizers and manure spreading and (iv) atmospheric deposition. Our results show that the TMM we studied could be divided into four distinct groups: Group A) elements mainly directly coming from the underlying aquifer and the stream channel (As, Ba and V); Group B) elements mainly acquired from running waters ( $\mathrm{Al}$ and $\mathrm{La}$ ); Group $\mathrm{C}$ ) elements acquired to a lesser extent by running waters $(\mathrm{Cr}, \mathrm{Cu}, \mathrm{Ni}$, $\mathrm{Pb}, \mathrm{U}$ and $\mathrm{Zn}$ ), a behavior we observed for all watersheds except the Doulon and the Allier ones, which correspond to gneissic watersheds with a forested land cover $>80 \%$ (Figure $6 \mathrm{a}, \mathrm{b}$ ) and Group D) Cd, whose concentrations showed variations between the watersheds but a relative seasonal consistency between the two hydrological stages. As the concentrations measured in Group A did not increase during the period when topsoil leaching was contributing, $\mathrm{As}, \mathrm{Ba}$ and $\mathrm{V}$ can be assumed to be representative of the natural geochemical background. For elements in Group B, the strong Al/La relationship observed may result from the association of these two elements with humic substances leached from the soils, which enhances their solubility by forming complexes [85-88]. Due to the possible formation of phosphate-REE complexes [38,39], La can originate from both rock weathering and the spreading of mineral fertilizers [89,90]. For elements in Group C, a previous study estimated that in our study area, more than $50 \%$ of As and $\mathrm{Cr}$ and more than $75 \%$ of $\mathrm{Cu}, \mathrm{Ni}, \mathrm{Pb}$ and $\mathrm{Zn}$ in soils are released by animal manure and that more than $40 \%$ of the $\mathrm{Cd}$ comes from mineral fertilizers (ADEME, [91]). In our watersheds, the agriculture practice is mostly confined to extensive livestock farming. Therefore, mineral fertilizers are not heavily used, which may explain the low $\mathrm{Cd}$ concentrations recorded. The chemical composition of the agricultural soils in the study area is thus mostly impacted by livestock feeding, including the medical treatments the animals receive. In addition, atmospheric deposition has to be considered as a potential source for these elements. Unfortunately, our dataset cannot unambiguously identify the primary origin for the elements in Group C. The stability of the Cd concentrations (group D) for each watershed suggests that none of the potential emission sources is dominating nor that dilution affects its concentrations in the water. 
Isotope geochemistry has widely demonstrated its added value for tracking sources of metals, e.g., $\mathrm{Cd}, \mathrm{Cr}, \mathrm{Cu}, \mathrm{Pb}, \mathrm{U}$ and $\mathrm{Zn}$, in the environment (e.g., [92] and references therein). This is based on the fact that metal isotope ratios/compositions usually show specific signatures representative of the reservoir they are originating from [93,94]. However, processes such as oxidation/reduction, sorption, dissolution/precipitation reactions or biological assimilation can modify some of these isotope distributions and induce isotope fractionation (i.e., modify isotope ratios/compositions). This can make the identification of the corresponding emission source(s) more difficult. Still, some of these metal isotope systematics, especially $\mathrm{Pb}$, are not subject to isotope fractionation and can thus be directly linked to their source reservoir. However, the ability for $\mathrm{Pb}$ isotope ratios to discriminate parent lithologies highly depends on the type of facies in presence. Figure $6 \mathrm{~b}$ shows that for the Upper Loire Basin, the isotope distinction can mostly be made between stream waters draining granites (having the lowest ${ }^{207} \mathrm{~Pb} /{ }^{206} \mathrm{~Pb}$ and ${ }^{208} \mathrm{~Pb} /{ }^{206} \mathrm{~Pb}$ ratios) and those draining basalts (that yield more radiogenic isotope ratios). All other lithologies give dissolved $\mathrm{Pb}$ intermediate isotope signatures that may not be interpreted as representative of the lithology the water is interacting with.

\subsection{Trace Elements Geochemical Baseline}

The studied watersheds were carefully selected to represent the least polluted watersheds of the upper crystalline Loire River Basin. However, our results show that the distribution of most of the TMM is impacted by anthropogenic activities from both local (agriculture practices, but not road traffic) and long (atmospheric deposit) ranges. Only $\mathrm{As}, \mathrm{Ba}$ and $\mathrm{V}$ concentrations measured at the low water stage can be considered as representative of the natural geochemical background, but the main lithologies cannot be discriminated by specific signatures. For the other elements, while establishing constant values for the natural geochemical background is not feasible, individual baselines can still be defined at the time of the water quality assessment for a given area and period. These can then be used as reference values for further regulation monitoring.

\section{Conclusions}

For regulatory institutions, defining the geochemical background or natural baseline is of the utmost importance for detecting water contamination. With the example of 10 monolithologic watersheds selected in the Upper Loire Basin (France), we implemented a geochemical approach coupling water chemistry and the isotope ratios of dissolved $\mathrm{Pb}$ to better constrain the sources and dynamics of TMM in the surface waters. Our results demonstrate that because of the low water-rock interaction rate, the chemical signature of the surface waters is mostly regulated by the minerals' weatherability rather than by the available TMM stock. Moreover, while stream waters from watersheds with identical dominating lithologies can exhibit different chemical characteristics, those from watersheds having distinct crystalline lithologies can show similar chemical characteristics, as their dissolved TMM will derive from the weathering of the same minerals (i.e., pyroxene, amphibole, mica). This shows that the main lithological characteristics of a watershed do not control the concentrations of dissolved trace elements in stream waters. Ultimately, this highlights the need to define in the future natural baselines at the watershed scale rather than at the national or European level.

This study also shows the importance of considering river discharge when designing the sampling strategy of studies aiming at monitoring surface water quality, as for most trace elements, topsoil leaching can significantly contribute to and impact the TMM budget. The protocol we are proposing also allows a geochemical baseline for the head of a given watershed to be defined with the assurance of a minimum impact from anthropogenic activities. This is of particular importance, as after a certain distance from the spring, inputs from tributaries can overprint the signature of the head water. This implies that several baseline values may need to be defined downstream and, more importantly, demonstrates 
the inappropriateness of defining a regional baseline when the length of the considered river becomes important.

Supplementary Materials: The following are available online at https:/ /www.mdpi.com/article/10 $.3390 / w 13131845 /$ s1, Table S1. Geographic characteristics of sampling sites. Geographic coordinates are expressed according to the Lambert 93 projection. Site 1: outlet; higher number: spring; Site P: preparative survey if distinct, Table $\mathrm{S} 2$. Pb isotope ratios measured in stream waters and in sediment, Table S3. Geographic characteristics of sampling sites from previous studies. Geographic coordonates are expressed according to the Lambert 93 projection.

Author Contributions: Conceptualization, D.W. and N.G.; methodology, D.W., F.C., N.G.; validation, D.W., F.C. and N.G.; formal analysis, D.W., F.C. and N.G.; investigation, F.C., P.V., D.W., N.G.; resources, D.W., N.G.; data curation, D.W., N.G.; writing—original draft preparation, N.G., D.W., F.C.; writing - review and editing, N.G., D.W.; project administration, D.W. and N.G.; funding acquisition, D.W., N.G., X.B. All authors have read and agreed to the published version of the manuscript.

Funding: This research was funded by Agence de l'Eau Loire-Bretagne, (No. 140193801).

Institutional Review Board Statement: Not applicable.

Informed Consent Statement: Not applicable.

Data Availability Statement: Data sharing not applicable.

Acknowledgments: This work is part of the FongéLoire project supported by the Agence de l'Eau Loire-Bretagne. We are grateful to the SARM at the CRPG UMR 7358 in Nancy for their assistance with the analysis of trace element concentrations by ICP-MS. The authors also thank Romain Nigris, who was involved in the project during his MSc, for his assistance during the field campaigns and for running the $\mathrm{Pb}$ isotope analysis. We thank the reviewers for their comments, which greatly improved the manuscript.

Conflicts of Interest: The authors declare no conflict of interest.

\section{References}

1. European Council. Directive of the European Parliament and of the Council (2000/60/EC) Establishing a Framework of Community Action in the Field of Water Policy; European Council: Brussels, Belgium, 2000.

2. European Council. Directive of the European Parliament and of the Council of 12 August 2013 (2013/39/EC) Amending Directives 2000/60/EC and 2008/105/EC as Regards Priority Substances in the Field of Water Policy; European Council: Brussels, Belgium, 2013.

3. Qu, S.; Wu, W.; Nel, W.; Ji, J. The behavior of metals/metalloids during natural weathering: A systematic study of the monolithological watersheds in the upper Pearl River Basin, China. Sci. Total Environ. 2020, 708, 134572. [CrossRef] [PubMed]

4. Shaheen, S.M.; Rinklebe, J. Geochemical fractions of chromium, copper, and zinc and their vertical distribution in floodplain soil profiles along the Central Elbe River, Germany. Geoderma 2014, 228-229, 142-159. [CrossRef]

5. Chapela Lara, M.; Buss, H.L.; Pogge von Strandmann, P.A.E.; Schuessler, J.A.; Moore, O.W. The influence of critical zone processes on the Mg isotope budget in a tropical, highly weathered andesitic catchment. Geochim. Cosmochim. Acta 2017, 202, 77-100. [CrossRef]

6. Murozumi, M.; Chow, T.J.; Patterson, C.C. Chemical concentrations of pollutant lead aerosols, terrestrial dusts and sea-salts in Greenland and Antarctic snow strata. Geochim. Cosmochim. Acta 1969, 33, 1247-1294. [CrossRef]

7. Steinnes, E.; Friedland, A.J. Metal contamination of natural surface soils from long range atmospheric transport: Existing and missing knowledge. Environ. Rev. 2006, 14, 169-186. [CrossRef]

8. Cong, Z.Y.; Kang, S.C.; Zhang, Y.; Gao, S.; Wang, Z.; Liu, B.; Wan, X. New insights into trace element wet deposition in the Himalayas: Amounts, seasonal patterns, and implications. Environ. Sci. Pollut. Res. 2015, 22, 2735-2744. [CrossRef] [PubMed]

9. Bing, H.; Wu, Y.; Li, J.; Xiang, Z.; Luo, X.; Zhou, J.; Sun, H.; Zhang, G. Biomonitoring trace element contamination impacted by atmospheric deposition in China's remote mountains. Atmos. Res. 2019, 224, 30-41. [CrossRef]

10. Salminen, R.; Gregorauskiene, V. Considerations regarding the definition of a geochemical baseline of elements in the surficial materials in areas differing in basic geology. Appl. Geochem. 2000, 15, 647-653. [CrossRef]

11. Reimann, C.; Garrett, R.G. Geochemical background-concept and reality. Sci. Total Environ. 2005, 350, 12-27. [CrossRef] [PubMed]

12. Johnson, C.C.; Breward, N. G-BASE Geochemical Baseline Survey of the Environment., Commissioned Report, CR/04/016N; British Geological Survey: Nottinghamshire, UK, 2004.

13. Salminen, R.; Batista, M.J.; Bidovec, M. Geochemical Atlas of Europe, Part 1 Geological Survey of Finland Publication. 2006. Available online: www.gtk.fi/publ/ foregsatlas (accessed on 26 June 2021). 
14. Albanese, S.; De Vivo, B.; Lima, A.; Cicchella, D. Geochemical background and baseline values of toxic elements in stream sediments of Campania region (Italy). J. Geochem. Explor. 2007, 93, 21-34. [CrossRef]

15. Larrose, A.; Coynel, A.; Schäfer, J.; Blanc, G.; Massé, L.; Maneux, E. Assessing the current state of the Gironde Estuary by mapping priority contaminant distribution and risk potential in surface sediment. Appl. Geochem. 2010, 25, 1912-1923. [CrossRef]

16. Imrie, C.E.; Korre, A.; Munoz-Melendez, G.; Thornton, I.; Durucan, S. Application of factorial kriging analysis to the FOREGS European topsoil geochemistry database. Sci. Total. Environ. 2008, 393, 96-110. [CrossRef]

17. Reimann, C.; Fabian, K.; Birke, M.; Filzmoser, P.; Demetriades, A.; Négrel, P.; Oorts, K.; Matschullat, J.; de Caritat, P.; Albanese, S.; et al. GEMAS: Establishing geochemical background and threshold for 53 chemical elements in European agricultural soil. Appl. Geochem. 2018, 88, 302-318. [CrossRef]

18. DePaula, F.C.; Mozeto, A.A. Biogeochemical evolution of trace elements in a pristine watershed in the Brazilian southeastern coastal region. Appl. Geochem. 2001, 16, 1139-1151. [CrossRef]

19. Santos-Francés, F.; Martínez-Graña, A.M.; Rojo, P.A.; Sánchez, A.G. Geochemical Background and Baseline Values Determination and Spatial Distribution of Heavy Metal Pollution in Soils of the Andes Mountain Range (Cajamarca-Huancavelica, Peru). Int. J. Environ. Res. Public Health 2017, 14, 859. [CrossRef] [PubMed]

20. Gustavsson, N.; Loukola-Ruskeeniemi, K.; Tenhola, M. Evaluation of geochemical background levels around sulfide mines-A new statistical procedure with beanplots. Appl. Geochem. 2012, 27, 240-249. [CrossRef]

21. Kelepertzis, E.; Argyraki, A.; Daftsis, E. Geochemical signature of surface water and stream sediments of a miner-alized drainage basin at NE Chalkidiki, Greece: A pre-mining survey. J. Geochem. Explor. 2012, 114, 70-91. [CrossRef]

22. Levitan, D.M.; Schreiber, M.E.; Seal, R.R., II; Bodnar, R.J.; Aylor, J.G., Jr. Developing protocols for geochemical baseline studies: An example from the Coles Hill uranium deposit, Virginia, USA. Appl. Geochem. 2014, 43, 88-100. [CrossRef]

23. Chandesris, A.; Canal, J.; Bougon, N.; Coquery, M. Détermination du Fond Géochimique Pour les Métaux Dissous Dans les Eaux Continentals; Rapport final. Irstea. 65 p + Annexes; IRSTEA: Rennes, France, 2013; p. 231.

24. Sahoo, P.K.; Dall'Agnola, R.; Negreiros Salomão, G.; da Silva Ferreira Juniora, J.; Sousa Silva, M. High reso-lution hydrogeochemical survey and estimation of baseline concentrations of trace elements in surface water of the Ita-caiúnas River Basin, southeastern Amazonia: Implication for environmental studies. J. Geochem. Expl. 2019, 205, 106321. [CrossRef]

25. Pételet-Giraud, E.; Négrel, P.; Casanova, J. Tracing Mixings and Water-rock Interactions in the Loire River Basin (France): 818O- $82 \mathrm{H}$ and 87Sr/86Sr. Procedia Earth Planet. Sci. 2017, 17, 794-797. [CrossRef]

26. Cheng, Z.; Foland, K.A. Lead isotopes in tap water: Implications for Pb sources within a municipal water supply system. Appl. Geochem. 2005, 20, 353-365. [CrossRef]

27. Petelet-Giraud, E.; Luck, J.M.; Ben Othman, D.; Négrel, P. Dynamic scheme of water circulation in karstic aquifers as constrained by $\mathrm{Sr}$ and Pb isotopes. Application to the Hérault watershed, Southern France. Hydrogeol. J. 2003, 11, 560-573. [CrossRef]

28. Bohdalkova, L.; Novak, M.; Stepanova, M.; Fottova, M.; Chrastny, V.; Mikova, J.; Kubena, A. The Fate of Atmos-pherically Derived $\mathrm{Pb}$ in Central European Catchments: Insights from Spatial and Temporal Pollution Gradients and Pb Isotope Ratios. Environ. Sci. Technol. 2014, 48, 4336-4343. [CrossRef]

29. Négrel, P.; Millot, R.; Roy, S.; Guerrot, C.; Pauwels, H. Lead isotopes in groundwater as an indicator of water-rock interaction (Masheshwaram catchment, Andhra Pradesh, India). Chem. Geol. 2010, 274, 136-148. [CrossRef]

30. Longman, J.; Veres, D.; Ersek, V.; Phillips, D.L.; Chauvel, C.; Tamas, C.G. Quantitative assessment of Pb sources in isotopic mixtures using a Bayesian mixing model. Sci. Rep. 2018, 8, 6154. [CrossRef]

31. Buffle, J.; De Vitre, R.R. (Eds.) Chemical and Biological Regulation of Aquatic Systems; Lewis Publishers; CRC Press Inc.: Boca Raton, FL, USA, 1994; p. 393.

32. Regenspurg, S.; Margot-Roquier, C.; Harfouche, M.; Froidevaux, P.; Steinmann, P.; Junier, P.; Bernier-Latmani, R. Speciation of naturally-accumulated uranium in an organic-rich soil of an alpine region (Switzerland). Geochim. Cosmochim. Acta 2010, 74, 2082-2098. [CrossRef]

33. Pili, E.; Tisserand, D.; Bureau, S. Origin, mobility, and temporal evolution of arsenic from a low-contamination catchment in Alpine crystalline rocks. J. Hazard. Mater. 2013, 262, 887-895. [CrossRef]

34. Illuminati, S.; Annibaldi, A.; Truzzi, C.; Tercier-Waeber, M.-L.; Nöel, S.; Braungardt, C.B.; Achterberg, E.P.; Howell, K.A.; Turner, D.; Marini, M.; et al. In-situ trace metal $(\mathrm{Cd}, \mathrm{Pb}, \mathrm{Cu})$ speciation along the Po River plume (Northern Adriatic Sea) using submersible systems. Mar. Chem. 2019, 212, 47-63. [CrossRef]

35. Pelfrêne, A.; Gassama, N.; Grimaud, D. Mobility of major-, minor- and trace elements in solutions of a planosolic soil: Distribution and controlling factors. Appl. Geochem. 2009, 24, 96-105. [CrossRef]

36. N'Guessan, Y.; Probst, J.; Bur, T.; Probst, A. Trace elements in stream bed sediments from agricultural catchments (Gascogne region, S-W France): Where do they come from? Sci. Total. Environ. 2009, 407, 2939-2952. [CrossRef] [PubMed]

37. Fan, Q.; Tanaka, M.; Tanaka, K.; Sakaguchi, A.; Takahashi, Y. An EXAFS study on the effects of natural organic matter and the expandability of clay minerals on cesium adsorption and mobility. Geochim. Cosmochim. Acta 2014, 135, 49-65. [CrossRef]

38. Banfield, J.P.; Eggleton, R.A. Apatite replacement and rare earth mobilization, fractionation, and fixation during weathering. Clays Clay Miner. 1989, 37, 113-127. [CrossRef]

39. Aubert, D.; Stille, P.; Probst, A. REE fractionation during granite weathering and removal by waters and suspended loads: Sr and Nd isotopic evidence. Geochim. Cosmochim. Acta 2001, 65, 387-406. [CrossRef] 
40. Costa, L.; Mirlean, N.; Johannesson, K.H. Rare earth elements as tracers of sediment contamination by fertilizer industries in Southern Brazil, Patos Lagoon Estuary. Appl. Geochem. 2021, 129, 104965. [CrossRef]

41. Chow, T.J. Pb accumulation in roadside soils and grass. Nature 1970, 225, 295-296. [CrossRef] [PubMed]

42. Cloquet, C.; Carignan, J.; Libourel, G.; Sterckeman, T.; Perdrix, E. Tracing Source Pollution in Soils Using Cadmium and Lead Isotopes. Environ. Sci. Technol. 2006, 40, 2525-2530. [CrossRef] [PubMed]

43. Komárek, M.; Ettler, V.; Chrastný, V.; Mihaljevič, M. Lead isotopes in environmental sciences: A review. Environ. Int. 2008, 34, 562-577. [CrossRef]

44. Criaud, A. Phénomènes D’oxydo-Réduction et Métaux en Traces dans les Eaux Minérales Carbogazeuses du Massif Central. Ph.D. Thesis, Denis Diderot University, Paris, France, 1983.

45. Gassama, N. Comportement du Nickel et du Cobalt dans des Eaux au Cours de Leur Évolution vers L'équilibre eau-Roche (zones granitiques). Ph.D. Thesis, Denis Diderot University, Paris, France, 1993.

46. Millot, R.; Desaulty, A.M. Projet ISOP-Recherche Méthodologique Pour L'identification des Sources de Polluants Métalliques sur le Bassin Loire-Bretagne; Rapport final; BRGM/RP-63717-FR; BRGM: Orléans, France, 2014; p. 79.

47. INSEE. 2015. 2008 and 2015 Census; INSEE: Paris, France, 2008.

48. CLC. CORINE Land Cover. Available online: https://www.data.gouv.fr/fr/datasets/corine-land-cover-occupation-des-sols-enfrance/ (accessed on 1 October 2014).

49. Basol. MEDDE. 2015. Available online: https://basol.developpement-durable.gouv.fr/recherche.php (accessed on 1 October 2014).

50. Pardo, I.; Poikane, S.; Bonne, W. Revision of the Consistency in Reference Criteria Application in the Phase I of the European INTERCALIBRATION Exercise, Rapport Final EUR 24843 EN-2011; JRC Scientific and Technical Report: Ispra, Italy, $2011 ;$ p. 94.

51. BRGM Infoterre. Available online: https:/ / infoterre.brgm.fr (accessed on 1 June 2015).

52. Hydro-MEDDE/De 2014, 2015, 2016. Banque Hydro. Available online: http:/ / www.hydro.eaufrance.fr (accessed on 1 June 2017).

53. INERIS. Normes de Qualité Environnementale et Valeurs Guides Environnementales, DRC-18-158732-03350A, Avril 2018; INERIS: Verneuil-en-Halatte, France, 2018.

54. European Council. Directive of the European Parliament and of the Council of (2015/1787/EC) Amending Directive 1998/83/EC on the Quality of Water Intended for Human Consumption; European Council: Brussels, Belgium, 2015.

55. Newman, K.; Georg, R.B. The measurement of Pb isotope rations in sub-ng quantities by fast scanning single col-lector sector field-ICP-MS. Chem. Geol. 2012, 304-305, 151-157. [CrossRef]

56. Manhès, G.; Minster, J.; Allègre, C. Comparative uranium-thorium-lead and rubidium-strontium study of the Saint Séverin amphoterite: Consequences for early solar system chronology. Earth Planet. Sci. Lett. 1978, 39, 14-24. [CrossRef]

57. Belshaw, N.; Freedman, P.; O’Nions, R.; Frank, M.; Guo, Y. A new variable dispersion double-focusing plasma mass spectrometer with performance illustrated for Pb isotopes. Int. J. Mass Spectrom. 1998, 181, 51-58. [CrossRef]

58. Woodhead, J. A simple method for obtaining highly accurate Pb isotope data by MC-ICP-MS. J. Anal. Spectrom. 2002, 17, 1381-1385. [CrossRef]

59. De Vitre, R.R.; Sulzberger, B.; Buffle, J. Transformations of Iron at Redox Boundaries. In Chemical and Biological Regulation of Aquatic Systems; Buffle, J., De Vitre, R.R., Eds.; Lewis Publishers: Boca Raton, FL, USA, 1994; pp. 91-130.

60. Stumm, W.; Morgan, J.J. Aquatic Chemistry: Chemical Equilibria and Rates in Natural Waters, 3rd ed.; Wiley-Interscience Publication: Hoboken, NJ, USA, 1996; p. 976.

61. Ball, J.W.; Nordstrom, D.K. WATEQ4F-User's Manual with Revised Thermodynamic Data Base and Test Cases for Calculating Speciation of Major, Trace and Redox Elements in Natural Waters; Open-File Report 90-129 for U.S. Geological Survey: Reston, VA, USA, 1991; p. 185.

62. Parkhurst, D.L.; Appelo, C.A.J. Description of Input and Examples for PHREEQC Version 3-A Computer Program for Speciation, Batch-Reaction, One-Dimensional Transport, and Inverse Geochemical Calculations: U.S. Geological Survey Techniques and Methods, book 6, chap. A43; U.S. Geological Survey: Reston, VA, USA, 2013; p. 497.

63. U.S. Environmental Protection Agency. MINTEQA2/PRODEFA2, A Geochemical Assessment Model for Environ-Mental Systems-User Manual Supplement for Version 4.0; Revised September 1999; National Exposure Research Laboratory, Ecosystems Research Division: Athens, GA, USA, 1998; p. 76.

64. Sarazin, G.; Fouillac, C.; Michard, G. Etude de l'acquisition d'éléments dissous par les eaux de lessivage des roches granitiques sous climat tempéré. Geochim. Cosmochim. Acta 1976, 40, 1481-1486. [CrossRef]

65. Grimaud, D.; Beaucaire, C.; Michard, G. Modelling of the evolution of ground waters in a granite system at low temperature: The Stripa ground waters, Sweden. Appl. Geochem. 1990, 5, 515-525. [CrossRef]

66. Gassama, N.; Violette, S. Geochemical study of surface waters in mountain granitic area. The Iskar upper watershed: Massif of Rila, Bulgaria. Water Res. 1997, 31, 767-776. [CrossRef]

67. White, A.F.; Blum, A.E.; Bullen, T.D.; Vivit, D.V.; Schulz, M.; Fitzpatrick, J. The effect of temperature on experimental and natural chemical weathering rates of granitoid rocks. Geochim. Cosmochim. Acta 1999, 63, 3277-3291. [CrossRef]

68. Sung, W.; Morgan, J.J. Kinetics and product of ferrous iron oxygenation in aqueous systems. Environ. Sci. Technol. 1980, 14, 561-568. [CrossRef]

69. Davis, S.H.R.; Morgan, J.J. Manganese (II) oxidation kinetics on metal oxide surfaces. J. Colloid Interface Sci. 1988, $129,63-77$. [CrossRef] 
70. Learman, D.; Wankel, S.; Webb, S.; Martinez, N.; Madden, A.; Hansel, C. Coupled biotic-abiotic Mn(II) oxidation pathway mediates the formation and structural evolution of biogenic Mn oxides. Geochim. Cosmochim. Acta 2011, 75, 6048-6063. [CrossRef]

71. Kabata-Pendias, A. Trace Elements in Soils and Plants, 4th ed.; CRC Press: Boca Raton, FL, USA, 2010; p. 550.

72. Xue, H.B.; Sigg, L.; Gaechter, R. Transport of Cu, Zn and Cd in a small agricultural catchment. Water Res. 2000, 34, 2558-2568. [CrossRef]

73. Aldrich, A.P.; Kistler, D.; Sigg, L. Speciation of $\mathrm{Cu}$ and $\mathrm{Zn}$ in Drainage Water from Agricultural Soils. Environ. Sci. Technol. 2002, 36, 4824-4830. [CrossRef]

74. Walraven, N.; van Gaans, P.; van der Veer, G.; van Os, B.; Klaver, G.; Vriend, S.; Middelburg, J.; Davies, G. Tracing diffuse anthropogenic $\mathrm{Pb}$ sources in rural soils by means of Pb isotope analysis. Appl. Geochem. 2013, 37, 242-257. [CrossRef]

75. Vilomet, J.D.; Veron, A.; Ambrosi, J.P.; Moustier, S.; Bottero, J.Y.; Chatelet-Snidaro, L. Isotopic Tracing of Landfill Leachates and Pollutant Lead Mobility in Soil and Groundwater. Environ. Sci. Technol. 2003, 37, 4586-4591. [CrossRef] [PubMed]

76. Roy, S.; Négrel, P. A Pb isotope and trace element study of rainwater from the Massif Central (France). Sci. Total. Environ. 2001, 277, 225-239. [CrossRef]

77. Negrel, P.; Guerrot, C.; Millot, R. Chemical and strontium isotope characterization of rainwater in France: Influence of sources and hydrogeochemical implications. Isot. Environ. Health Stud. 2007, 43, 179-196. [CrossRef] [PubMed]

78. Véron, A.; Flament, P.; Bertho, M.L.; Alleman, L.; Flegal, R.; Hamelin, B. Isotopic evidence of pollutant lead sources in Northwestern France. Atmos. Environ. 1999, 33, 3377-3388. [CrossRef]

79. Widory, D.; Roy, S.; Le Moullec, Y.; Goupil, G.; Cocherie, A.; Guerrot, C. The origin of atmospheric particles in Paris: A view through carbon and lead isotopes. Atmos. Environ. 2004, 38, 953-961. [CrossRef]

80. Resongles, E.; Dietze, V.; Green, D.; Harrison, R.; Ochoa-Gonzalez, R.; Tremper, A.J.; Weiss, D.J. Evidence for the continued contribution of lead deposited during the 20th century to the atmospheric environment in London of today. Proc. Natl. Acad. Sci. USA 2021. [CrossRef]

81. Klein, C.; Philpotts, A. Earth Materials: Introduction to Mineralogy and Petrology; Cambridge University Press: Cambridge, UK, 2013; p. 553.

82. Fourcade, S.; Allègre, C.J. Trace elements behavior in granite genesis: A case study The calc-alkaline plutonic asso-ciation from the Querigut Complex (Pyrénées, France). Contrib. Mineral. Petrol. 1981, 76, 177-195. [CrossRef]

83. Kemp, A.I.S.; Hawkesworth, C.J. Granitic Perspectives on the generation and secular evolution of the Continental Crust. In Treatise on Geochemistry, 1st ed.; Turekian, K.K., Holland, H.D., Eds.; Elsevier Science: Amsterdam, The Netherlands, 2003; pp. 349-410.

84. Kostitsyn, Y.A.; Volkov, V.N.; Zhuravlev, D.Z. Trace elements and evolution of granite melt as exemplified by the Raumid Pluton, Southern Pamirs. Geochem. Int. 2007, 45, 971-982. [CrossRef]

85. Viers, J.; Dupré, B.; Polvé, M.; Schott, J.; Dandurand, J.-L.; Braun, J.J. Chemical weathering in the drainage basin of a tropical watershed (Nsimi-Zoetele site, Cameroon): Comparison between organic-poor and organic-rich waters. Chem. Geol. 1997, 140, 181-206. [CrossRef]

86. Dia, A.; Gruau, G.; Olivié-Lauquet, G.; Riou, C.; Molénat, J.; Curmi, P. The distribution of rare earth elements in groundwaters: Assessing the role of source-rock composition, redox changes and colloidal particles. Geochim. Cosmochim. Acta 2000, 64, 4131-4151. [CrossRef]

87. Tang, J.; Johannesson, K.H. Speciation of rare earth elements in natural terrestrial waters: Assessing the role of dissolved organic matter from the modeling approach. Geochim. Cosmochim. Acta 2003, 67, 2321-2339. [CrossRef]

88. Johannesson, K.H.; Tang, J.; Daniels, J.M.; Bounds, W.J.; Burdige, D.J. Rare earth element concentrations and speci-ation in organic-rich blackwaters of the Great Dismal Swamp, Virginia, USA. Chem. Geol. 2004, 209, 271-294. [CrossRef]

89. Hu, Y.; Vanhaecke, F.; Moens, L.; Dams, R.; del Castilho, P.; Japenga, J. Determination of the aqua regia soluble content of rare earth elements in fertilizer, animal fodder phosphate and manure samples using inductively coupled plasma mass spectrometry. Anal. Chim. Acta 1998, 373, 95-105. [CrossRef]

90. Silva, F.B.V.; Nascimento, C.W.A.; Alvarez, A.M.; Araújo, P.R.M. Inputs of rare earth elements in Brazilian agri-cultural soils via P-containing fertilizers and soil correctives. J. Environ. Manag. 2019, 232, 90-96. [CrossRef]

91. ADEME. Bilan des Flux de Contaminants Entrant sur les sols Agricoles de France Métropolitaine. Deportes, I.; Départ. Gestion Biologique et Sols; ADEME: Angers, France, 2007; p. 330.

92. Wiederhold, J. Metal Stable Isotope Signatures as Tracers in Environmental Geochemistry. Environ. Sci. Technol. 2015, 49, 2606-2624. [CrossRef] [PubMed]

93. Rosca, C.; Schoenberg, R.; Tomlinson, E.; Kamber, B. Combined zinc-lead isotope and trace-metal assessment of recent atmospheric pollution sources recorded in Irish peatlands. Sci. Total. Environ. 2019, 658, 234-249. [CrossRef]

94. Zhong, Q.; Zhou, Y.; Tsang, D.; Liu, J.; Yang, X.; Yin, M.; Wu, S.; Wang, J.; Xiao, T.; Zhang, Z. Cadmium isotopes as tracers in environmental studies: A review. Sci. Total. Environ. 2020, 736, 139585. [CrossRef] [PubMed] 